\title{
Understanding the Reactivity of Metallic Nanoparticles: Beyond the Extended Surface Model for Catalysis
}

\author{
Francesc Viñes, ${ }^{1}$ José R. B. Gomes ${ }^{2}$ and Francesc Illas, ${ }^{1, *}$ \\ 1) Departament de Química Física \& Institut de Química Teòrica i Computacional \\ (IQTCUB), Universitat de Barcelona, C/Martí i Franquès 1, 08028 Barcelona, Spain. \\ ${ }^{2)}$ CICECO, Department of Chemistry, University of Aveiro, Campus Universitário \\ de Santiago, 3810-193 Aveiro, Portugal
}

\begin{abstract}
.-
Metallic nanoparticles constitute a new class of chemical objects which are present in different field as diverse as plasmonics, optics, catalysis or biochemistry. The atomic structure of the nanoparticle and its size usually determine the chemical reactivity but this is often masked by the presence of capping agents, solvent or supports. The knowledge of the structure and reactivity of isolated nanoparticles is a requirement when aiming at designing nanoparticles with a well-defined chemistry. Theoretical models together to computational chemistry efficient algorithms and parallel computer codes offer the opportunity to explore the chemistry of these interesting objects and to isolate size and shape effects allowing deriving some general trends.
\end{abstract}




\section{Introduction}

Metallic nanoparticles are ubiquitous in many areas of science, from physics to chemistry, materials science, and nanotechnology. From the point of view of fundamental physics, metallic nanoparticles provide models to explore effects of quantum confinement on optical, electronic, magnetic, and related properties. ${ }^{1,2}$ The interest of metallic nanoparticles in chemistry is related to the size and shape effect on their reactivity. The technologies related to these particles have direct impact in society and industry since, for instance, they are key components of supported heterogeneous catalysts ${ }^{3-5}$ working in environments as different as car exhausts, reactors in oil refineries, or in advanced photocatalytic processes. ${ }^{6}$ In the past few years, metallic nanoparticles have received increased attention because nanotechnology has opened the door to the synthesis of metallic nanoparticles with controlled size and shape, ${ }^{7-10}$ and because of the emerging applications in photonics, ${ }^{11-13}$ sensing, ${ }^{12,14-17}$ imaging, ${ }^{18,19}$ and even in medicine. ${ }^{20-23}$ In particular, the study of the chemical and physical properties of solely Au nanoparticles has become a blooming research field in itself with hundreds of articles published in the past few years. $^{24,25}$ Here it is worth to point out that the literature on the field, especially regarding experiments and applications, is extremely immense, and the references provided are aimed to guide the reader by providing appropriate initial steps.

Understanding the intimate chemistry of metallic nanoparticles is not a simple task since several factors are into play, such the above-commented size and shape, but also the composition. ${ }^{26}$ These have to be taken into account and, ideally, decoupled. In catalysis, metallic nanoparticles are usually deposited onto a support, most often an oxide or a sulfide. ${ }^{5}$ These systems are rather complex and experimental models have been proposed to control some of the parameters defining a supported catalyst by means of surface science techniques. ${ }^{27-30}$ Nevertheless, the chemistry of metallic clusters on homogeneous phase has also been the object of considerable attention since it is under these conditions that size and shape control is more easily achievable. ${ }^{31-33}$ However, it is important to realize that, except in the case of gas phase clusters concerning only up to a few tens of atoms, ${ }^{34}$ metallic particles in homogeneous phase are neither isolated nor clean, i.e., they are commonly found in colloidal form and stabilized by solvent molecules, polymers, microemulsions, 
sol-gel, or clays. ${ }^{31}$ The presence of the solvent and of the stabilizing agents renders the structural determination cumbersome.

Interestingly, back in 1995 it was possible to resolve the structure of $\mathrm{Pd}$ nanoparticles of different size stabilized in solution in the presence of tetraalkylammonium salts by a combination of scanning tunnelling microscopy (STM) and high-resolution transmission electron microscopy (HRTEM). ${ }^{35}$ Progress in the experimental characterization of metallic nanoparticles has been made in the case of $\mathrm{Pt}$, where model nanoparticles were synthetized by lithography techniques and colloid chemistry. ${ }^{36}$ These synthetic methods lead to the formation of two-dimensional polymer-capped metal nanoparticle arrays, which can be characterized using various typical surface science techniques, such as X-ray photoelectron spectroscopy (XPS) and atomic force microscopy (AFM), either before or after chemical reactions take place. Precisely because of the presence of the capping agents, the application of STM to nanoparticles thus obtained faces some not yet resolved issues. ${ }^{37}$ The experimental technology reached a point where metallic nanoparticles can be readily synthesized and characterized even at the subnanometer scale. ${ }^{38}$ The use of wet chemistry approaches has also contributed to our understanding of heterogeneous catalysis. For instance, Turner et al. ${ }^{39}$ were able to prepare $\mathrm{Au} / \mathrm{SiO}_{2}$ catalysts where the $\mathrm{Au}$ nanoparticles were derived from 55-atom clusters $\left(\mathrm{Au}_{55}\right)$ and to study the selective oxidation of styrene with dioxygen without the presence of any oxidation initiator. However, even in such a controlled study, a distribution of Au nanoparticles was obtained, which prevented the assignment of the observed reactivity to a particular type of nanoparticles with well-defined size. Clearly, several challenges still exist concerning the intrinsic effect of size and shape on the chemical reactivity of metallic nanoparticles. Again, the presence of capping agents and/or supports introduces additional complexity that renders the analysis of the chemical reactivity and potential catalytic activity a complicated task.

The tremendous progress in the synthesis of metallic nanoparticles with controlled size and shape briefly outlined above does not yet permit one to obtain isolated and clean samples, clearly limiting our capability to understand all the occurring critical phenomena, a necessary step towards engineering nanoparticles à la carte for a given application. Computational modelling offers a useful and complementary approach to experiments since 
dealing with isolated, clean, metallic nanoparticles does not represent a problem, except for the computational resources, which become significant for quantum mechanical calculations concerning particles in the 1-10 nanometer range, easily involving hundreds to thousands of atoms. Still, approximate theoretical models have been designed for these large particles, which are based on classical interatomic potentials containing parameters either fitted to experimental material properties or to density functional calculations. ${ }^{40}$ Nevertheless, modern computational codes and parallel computers can make these otherwise unfeasible calculations possible. In fact, significant progress has been made in the field regarding the structural properties of metallic and bimetallic nanoparticles, ${ }^{41}$ especially regarding the search for global minima in the smallest ones and tackling the problem of multitude of isomers in the medium and large size particles, ${ }^{40}$ which becomes very relevant in the case of bimetallic nanoalloys.

The progress in modelling metallic nanoparticles with density functional methods has grown considerably in the last decade, after several important contributions which go well beyond the study of small gas phase clusters with only a few atoms. It is worth mentioning the strategy followed by Yudanov et al. ${ }^{42}$ consisting in using moderately large nanoparticles to simultaneously represent terrace sites of extended surfaces and low coordinated sites. In this way, it is possible to explore the chemistry of well-defined single crystal surfaces and of supported catalysis within the same model, a strategy which represents a leap forward with respect to models representing low coordinated sites by extended stepped surfaces. ${ }^{43}$ For instance, with this alternative strategy, it is possible to gather information about the role of the particle size and to define the so-called scalable regime, as well as to study convergence of relevant properties towards the bulk. ${ }^{44}$ The two concepts are related but different; the scalable regime is reached when properties such as interatomic distances or cohesive energy start to exhibit a linear trend with increasing the number of atoms in the nanoparticle. This is opposite to the behaviour commonly found in small nanoparticles, where non-monotonic trends are observed upon increasing the nuclearity: in this regime every single atom counts. Note, however, that in the scalable regime certain properties may vary so little with respect to the particle size that, for practical purposes, one may consider the value converged. This is precisely the case the of the calculated adsorption energy for a particular adsorbate on a given type of facet. Clearly, 
establishing the scalable regime is important since it allows one to extrapolate properties towards larger nanoparticles and to the bulk. On the other side Yudanov et al. ${ }^{42}$ have shown that the calculated adsorption energy of $\mathrm{CO}$ on (111) terrace sites of truncated octahedral $\mathrm{Pd}$ nanoparticles is converged for particles having about 80 atoms or more. Scalability and convergence becomes especially important regarding the chemical reactivity of the metallic nanoparticles, with obvious implications in catalysis, because otherwise extracting general rules governing such processes in a systematic way becomes impossible.

The main focus of the present review is precisely on the chemical reactivity of metallic nanoparticles with the aim of understanding the chemistry beyond these fascinating systems and, whenever possible, to provide a first step towards determining rules allowing making reliable predictions on the reactivity of unsupported particles. This constitutes a necessary gateway before attempting a rational, engineered modification of the reactivity of nanoparticles. Improvements over the catalytic performance of metallic catalysts are induced, for instance, by an active interplay between particle and support, as recently shown for the case of the water gas shift reaction by Pt nanoparticles in $\mathrm{Pt} / \mathrm{CeO}_{2}(111)$ and $\mathrm{Pt} / \mathrm{CeO}_{\mathrm{x}} / \mathrm{TiO}_{2}(110)$ catalysts $^{45}$ or by nanoalloying, taking advantage of bimetallic catalysts but at the nanoscale. ${ }^{41}$ The main hypothesis here is that a deeper understanding of the particularities of the chemistry of metallic nanoparticles will allow improving their catalytic properties.

\section{Building nanoparticle structural models}

Because nanotechnology has emerged as one of the most important fields in physics, chemistry, and life sciences, the term nanoparticle is used in a very broad and often misleading way. In fact, nowadays the term nanoparticle is used in such a way that it covers almost all possible ranges from small gas phase clusters to very large particles containing thousands of atoms, including also cluster models representing extended surfaces. Figure 1 displays a $\mathrm{Pt}_{8}$ cluster, a three layer $\mathrm{Pt}_{29}$ cluster model representation of $\mathrm{Pt}(100)$, and a $\mathrm{Pt}_{598}$ cubo-octahedral particle with (111) and (100) facets, and illustrates these three quite welldefined regimes. The above words of caution apply not only to metallic nanoparticles but to

nanoparticles in the most general sense. ${ }^{46}$ Therefore a brief description of the different contexts is necessary to avoid misunderstandings and to be able to better focus the goal of the present review. 
Small clusters, such as that depicted in Figure 1a, have been extensively studied both at the experimental and theoretical levels. ${ }^{47}$ Experimental techniques such as seeded supersonic nozzle, gas aggregation, or laser vaporization, among others, are used as cluster sources. The cohesive energy, or binding energy per atom, strongly oscillates with the number of atoms, and the same occurs with other physical and chemical properties..$^{40,47}$ Several features of the electronic structure as well as some other properties of these systems, especially for simple metals, are quite well understood; the so-called shell model providing a quite unifying picture. Nevertheless, it is important to point out that the success of this simple model is a consequence of the boundary conditions of the nearly free electrons in small particles of simple metal clusters. ${ }^{47}$ Clearly, this simple model cannot account for the large variety of low lying isomers existing in a narrow energy range, which typically exhibit atomic structures hardly comparable to that of the bulk metal. The electronic structure of transition metal small clusters is a more complicated issue. ${ }^{40}$ Moreover, the chemistry of small metal clusters is by no means simple; the adsorption energies of probe molecules such as $\mathrm{CO}$ or NO strongly oscillate with cluster size. These systems are representative of the non-scalable regime defined in the previous subsection, and should not be referred to as nanoparticles but rather as metal clusters. Consequently, this type of systems will not be lengthy discussed here and for more exhaustive information the reader is referred to excellent reviews on the field. ${ }^{40,41,47}$

For completeness, it is worth mentioning that metal clusters containing a limited number of atoms have been broadly employed as surface models, especially in the 15 years spanning the 1980-1995 period. ${ }^{48,49}$ In this approach, the structure of the cluster is cut from the bulk and usually maintained fixed with the crystallographic parameters taken from experiment (Figure 1b). This choice, especially for small clusters, results in significant edge effects which, in turn, lead to a slow convergence of calculated results with respect to the cluster size. Precisely because of the edge effects, surface cluster models have been almost abandoned and substituted by periodic approaches, even if clusters have been very useful to unravel physical mechanisms related to local properties of adsorbate-surface interactions. ${ }^{49}$ The use of periodic surface models has been boosted by the appearance of quite accurate density functional theory based methods (see next section) and their implementation in efficient codes fully exploiting the translational symmetry. Cluster models of extended 
metallic surfaces may offer some advantages over the usual periodic slab model approach which, as already commented, has become the standard model in the theoretical study of surface chemistry and of the mechanisms of heterogeneously catalysed reactions. For instance, cluster models allow one to employ explicitly correlated wave function based methods which, when necessary, can be used to check the performance of a given density functional theory based method. Clearly, surface cluster models can be regarded as metallic nanoparticles, but the choice of a particular cluster shape strongly determines their physical and chemical properties.

We come now to the type of metallic particles which can be effectively denoted as nanoparticles; Figure 1c provides a prototypical example. These may contain from a few tens to several thousand of atoms, have dimension within 1-100 nm and usually exhibit very well defined crystal facets. Precisely because of this particular feature, it is common practice to build nanoparticle models using the well-known Wulff construction ${ }^{50}$ which was proposed as early as 1901 . The physical basis behind the Wulff construction is quite simple and follows from the fact that the equilibrium shape of a crystal is necessarily that minimising the total surface Gibbs energy. The nanoparticles built following this simple approach constitute a first approximate structural model, which can be refined in subsequent steps by energy minimization, using an appropriate method to estimate the total energy of the particle, as it will be briefly discussed in the next section.

The Wulff constructions may be viewed as a top-down approach since it starts from the knowledge of the surface energy $\gamma(h k l)$ of the exposed facets, which normally depend on the type of particle to be modelled. Usually one wishes to represent nanoparticles exhibiting most stable surfaces but, depending on the type of study, it may be convenient to include more reactive surfaces. For a given application, a good strategy is to mimic the type of nanoparticles which are revealed by TEM, a technique that permits to obtain structural information of supported nanoparticles even under realistic working conditions. ${ }^{51}$ Once the morphology of the nanoparticles of interest is defined, one needs to compute the set of surface energy $\gamma(h k l)$ values. This is a simple, standard, and almost routine calculation when employing appropriate slab models. Since the total Gibbs energy of a surface is given by the product of the surface energy $\gamma$ by the surface area $A$, one can assume that the equilibrium structure for a nanoparticle exposing facets $\{h k l\}$ Miller indexes is such that 


$$
\int \gamma(h k l) d A(h k l)=\text { minimum }
$$

where the integral involves all exposed facets; equation (1) is often referred to as the Wulff theorem. Wulff also proved that if $\gamma(\mathrm{hkl})$ is the surface energy of one of the exposed facets with these Miller indexes and $h(h k l)$ is the perpendicular distance from the surface to a point in the crystal known as Wulff's point, then for each of the exposed facets, the $\gamma(h k l) / h(h k l)$ ratio is constant. This allows one to construct the approximate equilibrium shape of the crystal following a simple recipe. The procedure, illustrated in Figure 2, starts from an arbitrary point of the perfect crystal and draws vectors normal to the crystallographic planes of interest. Next, from an arbitrary $\gamma(h k l) / h(h k l)$ ratio, mark the distances $h(h k l)$ to each one of the exposed $\{h k l\}$ surfaces along each of the vectors normal to $(h k l)$ drawn in the previous step. The desired nanoparticle equilibrium shape is now simply obtained by adding planes perpendicular to the vectors through the marked points and taking the intersection of the planes. A more detailed description can be found in the excellent book of Desjonqueres and Spanjaard about surface physics. ${ }^{52}$ It is also important to point out that more elaborate theoretical methods have been proposed to improve the original Wulff construction for isolated nanoparticles. The approach designed by Barnard and $Z_{\text {apol }}^{53}$ includes edge and corner energies, surface tensions, the bulk elastic energy and, in principle, can take into account experimentally relevant conditions such as surface composition or temperature.

The Wulff construction allows one to construct nanoparticle models with tailored facets, which can be used to study surface reactivity. Nevertheless, this constitutes only a starting point, and the final structure needs to be obtained by more elaborate first principles calculations as described in the next section. We must warn that the required calculations may be computationally very expensive or even unfeasible. Here it is where the concept of scalability defined in the introduction becomes tremendously useful. For very large nanoparticles it may well be that structural, physical, and chemical properties can be predicted from the values computed for smaller but morphologically similar nanoparticles. This is possible when the smaller nanoparticles are already in the scalable regime, i.e. range of particle sizes where properties of interest converge linearly to those of the bulk material as schematically illustrated in Figure 3. Nevertheless, one must warn that not all properties will enter the scalable regime at the same size and, therefore, finding the onset for this 
regime and checking that it is fulfilled for the property under scrutiny is crucial. The concept of scalable regime is fundamental for modelling purposes since it allows one to estimate properties of very large nanoparticles from appropriate smaller equivalent ones.

\section{Computational methods for metallic nanoparticles and their chemical reactivity}

The interest in metallic nanoparticles and clusters triggered several approaches, which are particularly well suited to explain experimental observations, such as the abundance and distribution of alkali clusters in the gas phase. The most popular method is based in the so-called shell model where a cluster is represented as a super-atom, with electrons fully delocalized in the cluster volume filling discrete energy levels. There are several implementations of increasing accuracy for this model. In the simplest one, the electronic structure is represented by a spherical Jellium model, which is similar to that used by Kohn and Lang in their seminal work leading to the interpretation of the surface work function in terms of a surface dipole (see for instance Ref. 52 and references therein). The spherical Jellium model also assumes that the electron density moves in a uniform background of opposite positive charge but, in addition, include an external potential. There are several forms of the potential, the simplest ones being the infinitely deep spherical and the harmonic well. The one electron Schrödinger equation for these simple models can be analytically solved and provide a series of levels, which allow to understand the series of magic numbers observed in the experiments. Note, however, that this simple model hampers the study of bond breaking and bond forming at the metallic nanoparticle surface. Therefore, a more detailed description of the shell model and other more or less empirical methods based in the use of some sort of predefined potentials is out of the scope of the present work, and the reader is referred to some of the excellent reviews in the subject. ${ }^{40,41}$

In order to study the reactivity of metallic nanoparticles (or of any system) toward a specific reaction, it is necessary to explore the potential energy surface and to be able to characterize reactants, product, and transitions states for the elementary steps of interest. This irremediably implies the use of quantum mechanical methods of electronic structure and also implies the need of considerable computational resources. The methods of electronic structure can be divided in two main families: in the first one, the goal is to find a suitable, sufficiently accurate, approximation to the $\mathrm{N}$-electron wave function, $\mathrm{N}$ being the total number of electrons in the system, whereas, in the second one, the aim is to extract the 
energy of the system and related properties from the electron density through an appropriate density functional; this is the basis of the so-called density functional theory (DFT) methods. An exhaustive description of the different methods of electronic structure requires several volumes and even a brief, schematic, explanation is out of the scope of the present review article and the interested reader is addressed to the available excellent textbooks on quantum chemistry or on electronic structure. Here, it is enough to comment that wave function based methods become rapidly computationally too expensive with $\mathrm{N}$ to be useful to explore the electronic structure and reactivity of metallic nanoparticles, whereas DFT based methods offer some hope and are nowadays the weapon of choice in this type of studies although these are not free of problems.

In order to understand the computational difficulties mentioned above it is sufficient to state that, even at the simplest Hartree-Fock level, where the N-electron wave function is represented by a single Slater determinant with orbitals usually expanded in a Gaussian type orbital (GTO) basis set and, hence, electron-electron interactions accounted for in an averaged way, the computational effort is unaffordable since the calculation of the necessary two-electron integrals typically imply a $\mathrm{N}^{4}$ dependence of the computational time. The number of electrons explicitly treated can be reduced by including the effect of the core electrons in an effective core potential (ECP) or other similar approaches, usually referred to as pseudopotentials. The use of ECPs is now a routine in calculations involving heavy elements, and has the advantage of including scalar relativistic effects, which are mainly due to the high kinetic energy of core electrons. An additional problem with the Hartree-Fock method is that it lacks electron correlation effects arising from the instantaneous electron-electron interaction, which are crucial when bonds are broken or formed. The simplest way to include electron correlation effects is to take it into account by second order perturbation theory. The description of the system is improved but still faces problems when interatomic distances are elongated and, to make it worse, the computational effort scales as $\mathrm{N}^{6}$. The computational burden increases if, in addition to the total energy, one needs to compute the energy gradients, a necessary requirement when aiming at finding optimum or transition state structures. Such structures have to be characterized, respectively, as minima or first-order saddle points in the potential energy surface, which requires additional frequency calculations. The latter calculations are 
unavoidable if the interest is on the chemical reactivity of nanoparticles, the main focus of the present work. The use of linear scaling techniques and of those based in the resolutions of the identity allows one to carry out total energy calculations within these methods at a lower cost, but this is still excessive for a small nanoparticle, i.e., a particle containing $\sim 100-200$ atoms.

Density functional theory based methods also make use of a single Slater determinant but here to represent the electron density which, as in the Hartree-Fock methods, is given by a set of orbitals expanded in some sort of basis set. However, at variance of Hartree-Fock, in DFT based methods the total energy is not calculated as variational expectation value of the exact non relativistic N-electron Hamiltonian but variationaly extracted from an appropriate functional acting on the electron density, known as the exchange-correlation functional. Hohenberg and Kohn proved that such functional exists although the exact form is not known. From the (unknown) exact functional one would obtain the ground-state exact energy and, hence, DFT based methods include electron correlation effects, the accuracy being provided by the choice of the functional. The simplest possible density functional is provided by the so-called local density approach (LDA), which assumes that the exchange-correlation potential depends on the density only. Including the density gradient in a specific, well determined, form leads to the generalized gradient approach (GGA), a family of functionals including the broadly used PW91 and PBE implementations. GGA functionals perform very well for metallic systems but are not as accurate for main chemistry elements. Mixing GGA exchange with non-local Fock exchange leads to the family of the so-called hybrid functionals, which are quite accurate for main chemistry elements. The interactions of adsorbates, normally containing main group elements, with simple or transition metals are usually well described with GGA functionals and, while accuracy is an unresolved issue, trends are usually correct. Finally, it is worth to point out that spin polarized density functional calculations can also be carried out when dealing with magnetic nanoparticles or with open shell adsorbates. Nevertheless, the treatment of open shells is not a trivial issue and the reader is referred to more specialized literature. For more details on the basics of DFT based methods and their application to transition metal containing systems of interest in Chemistry, the reader is addressed to the recent review paper by Cramer and Truhlar. ${ }^{54}$ 
Because DFT based methods only require the electron density, the computational burden goes as $\mathrm{N}^{3}$ and fully lineal schemes have also been proposed, which cannot be discussed here. This also implies a lower computational cost for energy gradients, geometry optimization, and transition state searches. Let us just add that the choice of the basis set needed to span the electron density is not a simple issue; GTO or Slater type orbitals (STO) are the normal choices although we will discuss below a convenient alternative. Nevertheless, DFT based calculations with GTO basis sets for quite large metallic nanoparticles have been reported in the pioneering work of Yudanov et al. ${ }^{42}$ These authors carried out DFT calculations for Pd nanoparticles explicitly including all electrons and scalar relativistic effects. These calculations became possible because of the efficient and smart use of point group symmetry, although this also implies the use of highly symmetrical models for the nanoparticles of interest; for instance, octahedral particles were considered in ref. 42. It is important to realize, however, that while the use of symmetry allows one to study rather large isolated nanoparticles, it becomes less efficient when considering the interaction of these particles with some adsorbate. One can still consider models where the presence of the adsorbate is replicated so as to keep the maximum symmetry. This strategy has also been followed by Yudanov et al. ${ }^{42}$ by considering for instance the adsorption of $\mathrm{CO}$ at the top of the 6 vertices of metallic nanoparticles with octahedral shape. This is, no doubt, a valid and clever approach but it becomes hardly useful when the problem of interest concerns an elementary reaction at the nanoparticle surface, as it will be shown below.

DFT based methods are very popular in solid state physics and surface science applications because of the relative facility to implement a periodic version of these methods, thus accounting for translational symmetry, which allows one to build realistic models of surfaces of solids. This is precisely the case when plane waves are used as basis sets to expand the electron density and it follows from the intrinsic periodicity of these functions. Plane waves describe the quantum state of a free particle in momentum space and a wave packet is necessary to avoid mathematical problems, as in the case of the wellknown Dirac delta function, which also describes the quantum state of a free particle but in the position space. Therefore, one may intuitively argue that these functions are not appropriate to describe the state of electrons in bound states. Note, however, that because of 
the imposition of periodic boundary conditions, plane waves fulfil the requirements of quantum mechanics, they are quadratically integrable and thus mathematically acceptable as basis functions; for a more elaborate discussion the reader is referred to the available textbooks in Quantum Mechanics. An appealing practical feature of plane wave basis sets is that the quality of the basis set, a delicate point and often a nightmare in quantum chemical calculations carried out with standard GTO or STO basis sets, is controlled by a single parameter, the kinetic energy cutoff controlling the number of plane waves in the calculations. In turn, the explicit description of the core electrons is difficult and requires an exceedingly large number of plane waves. Consequently, calculations with plane waves almost unavoidably use some sort of pseudopotential to represent the effect of the core electrons on the valence electron density. For a given accuracy, within a chosen density functional method, the number of plane waves is usually much larger than that number of GTO or STO basis functions but plane wave basis sets do not suffer from the well-known basis set superposition error characteristic of atom localized basis sets. The larger number of basis functions in the former case is not an issue since integrals involved are much faster when using plane waves. The latter offer also other additional computational advantages: the representation of the kinetic energy operator is diagonal, the energy derivatives are also computed in a faster way than when using localized basis sets, e.g. GTO or STO, and the overall procedure can be programmed in an efficient parallel fashion. Depending on the implementation, the number of iterations required to obtain a specific degree of accuracy becomes nearly independent of the system size. This is precisely the case of the widely used Vienna $\mathrm{Ab}$ initio Simulation Package $(V A S P)$ for which an efficient implementation reaches a scaling which is almost linear with the number of atoms for systems containing up to 1000 electrons or more. ${ }^{55}$ In the past few years, VASP has become a sort of standard in computational materials science although other schemes such as PWSCF, Quantum Espresso, or $A b$ Init are available. At this point the reader may wonder about the relationship of these solid state methods and the subject of this work, i.e., metallic nanoparticles. The answer is very simple, within the periodic implementation of DFT based methods is also possible to study finite, discrete, systems such as atoms or molecules, all that it is required is to place the system of interest inside a sufficiently large box which is periodically repeated. For a large enough periodic box, the interaction between the 
artificially replicated images is negligible and this can be controlled by increasing the dimensions of the box until convergence is reached. Here it is important to note that the larger the periodic box the higher the kinetic energy cutoff for the plane wave expansion, and the larger the number of plane waves to be included in the calculations. This is because of the need to fill the box space with a dense enough set of plane waves so that the electron density of the system can be accurately described.

The strategy outlined above has been tested by various authors on different types of metals including Fe, Pd, and Au (See Ref. 56 and references therein). Here we will focus on the work of Viñes et al. ${ }^{56}$ on a set of model Pd nanoparticles exhibiting cubo-octahedral symmetry of increasing size. The resulting $\mathrm{Pd}_{38}, \mathrm{Pd}_{44}, \mathrm{Pd}_{55}, \mathrm{Pd}_{79}, \mathrm{Pd}_{85}, \mathrm{Pd}_{116}, \mathrm{Pd}_{140}, \mathrm{Pd}_{146}$, and $\mathrm{Pd}_{225}$ nanoparticles include those used in the benchmark scalar relativistic DF calculations of Yudanov et al. ${ }^{42}$ and, hence, allowed Viñes et al. to establish the accuracy of the plane wave periodic approach. These authors used the average coordination number $\mathrm{N}_{\mathrm{av}}$, defined as a sum of the coordination numbers of all $\mathrm{n}$ atoms of a cluster divided by $\mathrm{n}$, and the fraction of surface atoms $n_{s} / n$ to characterize the surface/bulk features of these Pd nanoparticles, and have shown that these two parameters clearly exhibit the development of bulk character with increasing $\mathrm{n}$. Interestingly, the calculated values for these two parameters also reveal that a particle as large as $\mathrm{Pd}_{225}$ is still quite far from the bulk limit. Figure 4 reproduces the results of Viñes et al. ${ }^{56}$ for the scaling of the average Pd-Pd bond distance $d$ with the cluster size, and compares also with the all electron scalar relativistic calculations of Yudanov et al., ${ }^{42}$ which fully exploited point group symmetry. The emergence of the scalable regime is clear from the fitted correlation $\mathrm{d}=253.6+1.661 \mathrm{~N}_{\mathrm{av}}$ (in pm) which gives an extrapolated value for Pd bulk of $273.5 \mathrm{pm}$, very close to $272.7 \mathrm{pm}$ calculated value for bulk using the same density functional approach. Figure 4 also shows that the values obtained from the plane wave periodic approach are in very good agreement with the results of scalar relativistic cluster calculations performed using $\mathrm{O}_{\mathrm{h}}$ point-group symmetry, for which the fitted correlation was $d=253.1+1.667 \mathrm{~N}_{\mathrm{av}}$ and the distance extrapolated to Pd bulk was $273.1 \mathrm{pm} .{ }^{42}$ The average cohesive energy per atom is another important property providing information about convergence with respect to bulk and of whether the scalable regime has been reached or not. Usually, this property is represented as a function of the inverse of the mean particle radius approximated by $\mathrm{n}^{-1 / 3}$. ${ }^{42}$ The results 
of Viñes et al. $^{56}$ using the plane wave periodic approach are perfectly linear. The extrapolation to bulk Pd agrees quantitatively with the corresponding bulk values calculated explicitly. A final aspect to be considered regards the electronic structure of these Pd nanoparticles and how the bulk-like electronic properties are developed for metal nanoparticles of increasing size. The results of Viñes et al. ${ }^{56}$ evidence a relatively fast evolution of the density of states (DOS) towards the bulk Pd with increasing particle size; for particles larger than $\operatorname{Pd}_{79}$, the centre of the $d$-band, which is an excellent reactivity descriptor, approaches the bulk value.

Before closing this section it is important to comment on the electronic ground state of metallic nanoparticles with or without adsorbates. For nonmagnetic metals, the electronic ground state is often of closed shell type and it is possible to use a single Slater determinant to represent the electron density. However, one must warn that small clusters on nonmagnetic metals may exhibit an open shell ground state and one must carefully explore different electronic configurations to assure that the electronic ground state has been determined. The spin polarized version of DFT has to be used and one must be aware that the final density may not correspond to that of a state with well-defined multiplicity. It is even possible that the final electron density corresponds to a non-well defined mixing of different electronic states. This is indeed the usual situation when aiming at finding the electronic state of magnetic nanoparticles. Note also that the presence of adsorbates, with or without open shells, can further complicate the situation since it may well be that the presence of the adsorbate changes the electronic ground state of the metallic nanoparticle. Finally, note that support effects may also affect the electronic ground state of the supported particle, especially in the case of magnetic metals. In the forthcoming discussion we will be mainly concerned with closed shell systems, and appropriate caveats about this particular issue will be included when necessary.

To summarize, the electronic structure of metallic nanoparticles of moderate size can be studied by the present state-of-the-art density functional theory based methods. Localized or plane wave basis sets can be used to accurately describe the main features of these particles. Selected results suggest that particles containing 100-200 atoms exhibit properties which are significantly different from the bulk limit but also that beyond this size one can safely assume that the scalable regime has been reached and that, for larger 
nanoparticles, properties of interest such as cohesive energy (or adsorption energy of a given adsorbate as it will be discussed in the next section) can be safely extrapolated from the trends exhibited by smaller ones in this regime.

\section{Adsorption at metallic particles}

The reactivity of metallic nanoparticles for a specific reaction is intrinsically dependent on their size and shape and, therefore, it is tuneable. Adsorption has a crucial role in catalysis since it determines the binding strength and the location of reactants in the catalyst and how these diffuse, react on the surface until products eventually desorb. These chemical processes usually involve several steps through a variable number of transition states depending on the complexity of the reaction, i.e., depending on the number of elementary steps. Note that in some cases, the adsorption process is accompanied by catalyst surface reconstruction or refaceting. This intricate surface chemistry makes the experimental study of the adsorption process at metallic particles a difficult task. Hence, computational studies involving well-controlled conditions assume a pivotal role in this respect, despite the consideration of several approximations in order to make the calculations tractable.

As introduced above, Yudanov et al. ${ }^{42}$ considered symmetric (octahedral and cuboooctahedral) Pd nanoparticles and CO as a probe molecule, to investigate size effects on several calculated adsorbate/nanoparticle energetic and geometric properties. They described the structure of the nanoparticles either with a bulk-terminated geometry, where the Pd-Pd nearest-neighbour distances were fixed at the experimental value of Pd bulk, i.e., $2.750 \AA$, or with a fully optimized geometry within selected symmetry constraints, where the geometry of $\mathrm{Pd}$ nanoparticles, with sizes ranging from $\sim 1.1 \mathrm{~nm}\left(\mathrm{Pd}_{55}\right)$ to $\sim 1.9 \mathrm{~nm}$ $\left(\mathrm{Pd}_{146}\right)$, Figure 5 , is determined by a small number of symmetry-nonequivalent atoms (e.g. nine atoms in the case of the largest particles studied). In order to maintain the $\mathrm{O}_{\mathrm{h}}$ symmetry of the bare particles in the $\mathrm{CO}$ /nanoparticle calculations, a $\mathrm{CO}$ molecule was inserted at each (111) facet in the Pd nanoparticles, which corresponds to a total of eight $\mathrm{CO}$ molecules per particle. The interaction of $\mathrm{CO}$ with 3-fold hollow sites at the center of the (111) facets showed that with the exception of the smallest particle, i.e., $\operatorname{Pd}_{55}$, the calculations provided rather uniform interatomic distances, vibrational frequencies and adsorption energies for the remaining particles considered, i.e., $\operatorname{Pd}_{79}, \mathrm{Pd}_{85}, \mathrm{Pd}_{116}, \mathrm{Pd}_{140}$, and 
$\mathrm{Pd}_{146}$, Figure 5. In the latter, the adsorption energies calculated at the GGA-BP86 level of theory, including corrections for the basis set superposition error, were found to vary in a narrow interval (171 to $176 \mathrm{~kJ} / \mathrm{mol}$ ), the adsorbate to substrate distances were in the range 2.038 to $2.067 \AA$ and the $\mathrm{C}-\mathrm{O}$ vibrational mode varied between 1738 and $1755 \mathrm{~cm}^{-1}$. The calculated data for the smallest particle were $149 \mathrm{~kJ} / \mathrm{mol}, 2.017 \AA$ and $1711 \mathrm{~cm}^{-1}$, respectively. As it can be understood from these results, properties with a local character, e.g. bond lengths and frequencies, are not very different in the former and in the latter particles, but the adsorption energy on $\mathrm{Pd}_{55}$ is significantly smaller than those reported for the other five nanoparticles. This is due to the incomplete coordination featured by the three $\mathrm{Pd}$ atoms forming the hollow site in the case of $\mathrm{Pd}_{55}$. Thus, Yudanov et al. concluded that significant nanoparticle size effects are already eliminated for particles composed by about $80 \mathrm{Pd}$ atoms. In fact, from comparison of the interaction energies calculated for the largest Pd nanoparticles with the adsorption energies at 3-fold hollow sites, but on the extended $\operatorname{Pd}(111)$ surface, quantitative agreement was obtained. This seems to be a general conclusion if the same lattice constant and the same exchange-correlation functional are used for calculations involving nanoparticles with sizes $>\sim 100$ atoms or involving extended surfaces. Interestingly, the consideration of two other GGA functionals, namely, PW91 and RPBE, resulted in geometric parameters similar to those calculated with the BP86 functional for the largest Pd particles, but in quite different interaction energies, e.g. $\sim 176 \mathrm{~kJ} / \mathrm{mol}, 188 \mathrm{~kJ} / \mathrm{mol}$, and $142 \mathrm{~kJ} / \mathrm{mol}$ for BP86, PW91, and RPBE approaches, respectively.

Another important issue is the average metal-metal distance that is found to increase with the particle size. Yudanov et al. ${ }^{42}$ found that the consideration of relaxed nanoparticles (with Pd-Pd distances of $\sim 2.69 \AA$ or $2.65 \AA$, LDA approach, for the largest particles and for $\mathrm{Pd}_{55}$, respectively) resulted in the reduction of the $\mathrm{CO}$ adsorption energies by $\sim 10 \%(<20$ $\mathrm{kJ} / \mathrm{mol}$ ), in the case of the larger clusters, while a more significant reduction, $\sim 35 \mathrm{~kJ} / \mathrm{mol}$, was calculated for $\mathrm{Pd}_{55}$. The reduction in the binding energies agrees with the principle of bond order conservation as a result of better saturated valences in the nanoparticle Pd atoms. The variation in the calculated C-O and adsorbate-nanoparticle bond lengths was quite small but note that adsorbate induced reconstruction of the Pd nanoparticles was not considered. ${ }^{42}$ The consideration of a GGA approach was found to yield Pd-Pd average 
distances that are $\sim 0.1 \AA$ longer. Thus, from what has been said above, the choice of the DFT functional has an evident influence in the values of the calculated adsorbatenanoparticle energies; hence, it has a determining role in reactivity.

In a subsequent study, Yudanov et al. ${ }^{57}$ extended their DFT calculations (BP86 approach and GTO basis sets) to the interaction of $\mathrm{CO}$ with: bridge and top positions at the (111) facets; 4-fold hollow and top sites at (001) facets; bridge sites at particle edges; top sites at particle corners; and top sites above single Pd atoms deposited at the (111) facets using Pd nanoparticles with 116, 140, 146, 147, and $148 \mathrm{Pd}$ atoms, Figure 5, in most cases with Pd-Pd distances fixed at 2.750 ̊. In the case of the (111) and (001) facets, the relative strength of the adsorption positions found for the $\operatorname{Pd}(111)$ surface is maintained, i.e., the interaction energy increases in the order top $<$ bridge $<$ hollow sites. In fact, Yudanov et al. found that if the atoms forming the adsorption site have coordination number nine, the calculated properties are very similar to those calculated on the ideal $\operatorname{Pd}(111)$ surface. Although the geometries are similar, the interaction of $\mathrm{CO}$ with positions formed by low coordinated atoms is much stronger. Actually, the binding energy at top sites on corners is $\sim 30 \mathrm{~kJ} / \mathrm{mol}$ larger than at top positions in the middle of the facets; ${ }^{57}$ yet, the largest value for the interaction energy, $188 \mathrm{~kJ} / \mathrm{mol}$, was calculated for $\mathrm{CO}$ interacting with the bridge sites at the particle edges. The predicted effect of geometry relaxation on the energies calculated for $\mathrm{CO}$ interacting with low-coordinated atoms on large nanoparticles was suggested to be $\sim 10 \mathrm{~kJ} / \mathrm{mol}$, i.e., similar to that found for high-coordination sites.

Based on the calculated energetic data, the experimentally observed vibrations at low pressure for $\mathrm{CO}$ adsorbed on bridge and top positions of $\mathrm{Pd}$ nanoparticles containing approximately 400-3000 $\mathrm{Pd}$ atoms, deposited onto an alumina film, were assigned to adsorption on bridge sites at cluster edges and low-coordinated top positions, respectively. The experimental vibrations were obtained by infrared reflection absorption spectroscopy (IRAS) and sum frequency generation (SFG) techniques. ${ }^{57}$ Despite the difference in size of the Pd nanoparticles studied computationally and experimentally, the calculated energetic data agree well with the observation of experimental vibrations for $\mathrm{CO}$ at the top positions on small, defect-rich, Pd particles at pressures lower than on larger, defect-poor, Pd particles. 
The spectroscopic identification of the exposed adsorption sites on Pd nanoparticles was the aim of another combined computational (DFT) and experimental (IRAS) study but using nitric oxide as probe molecule. ${ }^{58}$ As in the study of Yudanov et al., ${ }^{57}$ the experimental study considered Pd nanoparticles supported by an alumina film deposited on NiAl(110) and the DFT calculations, considering either LDA or GGA approaches and unsupported cubo-octahedral Pd particles with 79, 116, and $140 \mathrm{Pd}$ atoms, were employed to interpret the experimental IR data monitored as a function of $\mathrm{NO}$ exposure in a temperature region between 100 and $300 \mathrm{~K}$. For comparison purposes, the calculations were also extended to NO adsorption on the single-crystal $\mathrm{Pd}(111)$ surface. Tests performed for the bare or NO covered Pd models showed that spin-polarization effects were not important. Thus, metal nanoparticles of non-magnetic elements behave to a large extent as the corresponding single-crystal metal surfaces, for which the effect of spin-polarization was suggested to be less important, i.e., the numerical differences in the calculated total energies and bond lengths corresponding to spin restricted or spin unrestricted formalisms are usually smaller than the inherent error of density functional theory based methods. ${ }^{59}$ The comparison of the results calculated with the different DFT approaches for NO interacting with $\operatorname{Pd}(111)$ showed that GGA Pd-Pd distances are longer than those calculated with LDA and also than the experimental ones but, very important, the GGA calculated N-O stretching vibration and the NO adsorption energy are in better agreement with the experimental data available. The agreement was notable $\left(<10 \mathrm{~cm}^{-1}\right.$ and $<5 \mathrm{~kJ} / \mathrm{mol}$, respectively) in the case of the RPBE approach. For NO interacting with two different sites on the three particles considered, calculations at the VWN level of theory suggested that a sufficient level of accuracy was already obtained with the smallest particle, since the changes in the $\mathrm{N}-\mathrm{O}$ vibration frequency and in the adsorption energy were smaller than $10 \mathrm{~cm}^{-1}$ and $20 \mathrm{~kJ} / \mathrm{mol}$, respectively. Therefore, Viñes et al. ${ }^{58}$ considered the RPBE approach and the $\mathrm{Pd}_{79}$ particle to perform a more detailed investigation on the interaction of $\mathrm{NO}$ with several different adsorption sites, e.g. top, bridge, and hollow sites on the (100) facets or on the (111) facets, corners, and edges positions. In such calculations, first and second Pd neighbours have been allowed to relax during geometry optimization. In the case of the $\mathrm{Pd}_{79}$ particle, adsorption was found to be preferred at 3-hollow sites (fcc preferred to hcp by $\sim 5 \mathrm{~kJ} / \mathrm{mol}$ ) followed closely by bridge sites on the edges. The quality of the calculations is demonstrated by the 
very good agreement between the calculated frequencies $\left(1525,1530\right.$ and $1651 \mathrm{~cm}^{-1}$ for fcc, hcp, and bridge at the edges sites, respectively) and the experimental low-coverage signals at $1520-1530 \mathrm{~cm}^{-1}$ and $1640-1645 \mathrm{~cm}^{-1}$. After the full occupation of these more favourable sites, and based on the calculated frequencies, it was suggested that on-top sites at the particle corners are occupied next.

In a more recent work, Yudanov et al. ${ }^{60}$ studied the size dependence of adsorption properties of metal nanoparticles based also on DFT energies for CO interacting with Pd particles containing between 13 and 116 atoms, i.e., they performed a detailed study on the non-scalable size regime for which deduction or extrapolation of properties is not possible. Note that particles with sizes in this region were suggested to be quite active for catalysis, so, understanding how the reactivity of small metal particles scales with size is fundamental for the design of new catalysts. As expected, since small particles present atoms with a higher variety of coordination numbers, strong variations were calculated for the adsorption energies of $\mathrm{CO}$ on the particles with sizes below $\sim 80 \mathrm{Pd}$ atoms. Nevertheless, the variation of the largest adsorption energies on going from the smaller to the largest Pd nanoparticles, Figure 6, shows two opposite trends: in the scalable regime, it is seen an increase of the binding strength with the diameter of the nanoparticles, which was suggested to be associated with an elongation of the average Pd-Pd distance caused by surface stress and a change in the van der Waals attraction (bonding competition); in the non-scalable regime, the binding strengths decreases with the size of the nanoparticles, which was associated to a shift of the centre of the $d$-band to larger binding energies with the increase of the particle size, in part as a consequence of a decreasing fraction of low coordinated atoms on the surface of the particle. The intersection of the two trends was found for particles with $\sim 50$ atoms for which the lowest energies for $\mathrm{CO}$ interacting with $\mathrm{Pd}$ nanoparticles were calculated. $^{60}$

The effects in the $\mathrm{CO}$ and $\mathrm{O}$ adsorption energies introduced by the increasing of the nanoparticle size was recently investigated with DFT by Kleis et al. ${ }^{61}$ using gold nanoparticles with nuclearities 13, 55, 147, 309, 561, 923, and 1415 atoms and having diameters between 0.8 and $3.7 \mathrm{~nm}$. The calculations were performed for fixed and relaxed geometries, the former with the adsorbate always at the same distance from the adsorption site in the different-sized particles to measure the electronic effects caused by varying the 
size of the particles. The general conclusion that the adsorption becomes stronger as the particle becomes smaller is still observed. The effects of relaxation in the adsorption energies for the smallest particles were found to be more important in the case of $O$ than of $\mathrm{CO}$ adsorption. In the case of $\mathrm{CO}$, the energy variations were found to be similar to those reported by Yudanov et al. for the interaction with Pd nanoparticles. ${ }^{42}$ In other words, the calculations show that the electronic effects totally dominate the relaxation effects, i.e., variations in the adsorption energies for frozen and relaxed calculations are smaller than those for different-sized clusters. Additionally, it is shown that even for the particle with 309 gold atoms, finite-size effects are still important, especially, in the case of $\mathrm{O}$ adsorption. Analyses of charge density difference plots between bare particles and particles interacting with an adsorbate show that the densities of gold atoms nearest-neighbouring and next nearest-neighbouring the adsorption sites are affected, i.e., gold atoms at the edges are involved in the adsorbate-nanoparticle interaction, which suggests that convergence to $\mathrm{Au}(111)$ of the adsorption energies calculated for the particle with 309 gold atoms was not achieved. It should be noted that these effects are more evident for $\mathrm{O}$ than for $\mathrm{CO}$ adsorption, which is possibly due to the stronger binding of the former to metal surfaces.

The interaction of another strongly bonded adsorbate, atomic carbon, with either Pd and Pt nanoparticles was reported in the literature. ${ }^{62,63}$ These studies were inspired by the observation that adsorbed atomic $\mathrm{C}$ species are formed in the course of surface reactions. These species commonly decorate late transition metal ( $\mathrm{Pt}, \mathrm{Pd}, \mathrm{Ni}, \mathrm{Rh}$, etc.) surfaces and constitute one of the major poisoning species of catalysts based on these metals, which are employed in a broad range of reactions dealing with organic molecules, e.g. steam and dry reforming, and partial oxidation. The calculations performed with the PARAGAUSS code by Neyman et al. ${ }^{62}$ were based on Pd particles with sizes ranging from $\sim 1.1 \mathrm{~nm}\left(\mathrm{Pd}_{55}\right)$ to $\sim 1.9 \mathrm{~nm}\left(\mathrm{Pd}_{146}\right)$, Figure 5, and show that $\mathrm{C}$ atoms are strongly adsorbed in the form of carbidic species that bear a significant negative charge. These authors found as well that the effect of spin polarization was quite marginal for the structure and energetics of bare and $\mathrm{C}$ covered palladium nanosize clusters. The changes in the cohesive energy and in the adsorption energy of $\mathrm{C}$ on threefold hollow sites of $\mathrm{Pd}_{85}$ are smaller than $1 \mathrm{~kJ} / \mathrm{mol}$ when spin polarization is taken into account. The calculated data for the adsorption of carbon at the most abundant hollow sites in the middle of the (111) facets, for particles with Pd-Pd 
distances frozen at $2.75 \AA$, show adsorbate to particles heights of $1.86 \pm 0.01 \AA$ (LDA), and adsorption energies between $624\left(\mathrm{Pd}_{55}\right)$ and $657\left(\mathrm{Pd}_{116}\right) \mathrm{kJ} / \mathrm{mol}(\mathrm{BP} 86)$, or between 643 $\left(\mathrm{Pd}_{55}\right)$ and $677\left(\mathrm{Pd}_{116}\right) \mathrm{kJ} / \mathrm{mol}(\mathrm{PW} 91)$. Relaxation of all degrees of freedom but restrained to the intrinsic $\mathrm{O}_{\mathrm{h}}$ symmetry of the models, introduces small changes in the Pd-Pd and C-Pd distances, and in the calculated adsorption energies. For instance, values of $2.70 \AA, 1.86 \AA$, and $670 \mathrm{~kJ} / \mathrm{mol}$ (BP86), respectively, were calculated for the $\mathrm{Pd}_{116}$ particle. Confirming conclusions from previous studies, convergence with particle size is faster for the distances than for the adsorption energies, and the relaxation contributions to the absolute values were found to be smaller than the effects introduced by different particle sizes. Interestingly, the calculations predict that the $\mathrm{C}$ atoms prefer to bind highly-coordinated sites (hollows in the middle of the (111) or, preferentially, of (100) facets), i.e., an important destabilization was found for $\mathrm{C}$ located at sites near edges of the (111) facets, which contrasts with the behaviour found for $\mathrm{CO}$ on Pd particles,${ }^{57}$ and with the results for the interaction of $\mathrm{C}$ atoms with Pt particles. ${ }^{63}$ In the latter case, Viñes et al., using the VASP code and the RPBE functional, found that the adsorption of carbon atoms seems to be enhanced near the particle edges. ${ }^{63}$ Very recently, Aleksandrov et al.$^{64}$ performed another computational work analysing the interaction of $\mathrm{C}$ atoms with $\mathrm{Pd}$ nanoparticles. In clear contrast with the results from the PARAGAUSS calculations of Neyman et al., ${ }^{62}$ the adsorption energies calculated with LDA, PW91, and RPBE approaches concerning the interaction of $\mathrm{C}$ species with sites near the facet edges is at least as favourable as with the hollow sites in the centre of the facets. This may be seen as another example of the important impact of the computational strategy in the calculated data for adsorbates interacting with metal particles.

Recently, some of us have been engaged in the computational study of the interaction of a single water molecule on a series of cubo-octahedral $\mathrm{Pt}_{\mathrm{n}}$ nanoparticles of increasing sizes $\left(\mathrm{n}=13,19,38,55,79\right.$, and 140) ${ }^{65}$ Very interestingly, the calculations indicate that the adsorption energy of water is not significantly influenced by the nanoparticle size, with energies of $52,45,47,57,48$, and $52 \mathrm{~kJ} / \mathrm{mol}$, respectively. These energies were calculated for water interacting with top sites at the particle edges (lowcoordinated sites) and, not surprisingly, are considerably larger than the value of $26 \mathrm{~kJ} / \mathrm{mol}$ calculated for water interacting with top sites on the extended $\operatorname{Pt}(111)$ surface. The quite 
converged interaction energies calculated for the water molecule on "computationally speaking" very small, small, and medium size Pt particles may be seen more as an exception than a rule. For instance, calculations with similar particles and computational parameters for $\mathrm{O}_{2}$ on $\mathrm{Au}_{\mathrm{n}}(\mathrm{n}=38,55,79,116$, and 147) nanoparticles originated much larger energy differences, more precisely, adsorption energies of 93, 22, 27, 53, and 34 $\mathrm{kJ} / \mathrm{mol}$, respectively. ${ }^{66}$

\section{Reactivity on metallic particles}

In this last section, a step forward is made going beyond the methodology suitability on metal nanoparticles adsorptive properties. The adequacy of the models and computational levels is proven by studying chemical reactivity on metallic nanoparticles, simulating model catalysts composed of supported metal nanoparticles on a given substrate, typically an metallic oxide. Below we show, for selected exemplary reactions catalysed by metal nanoparticles, how the reaction mechanism is dependent not only on the size and shape, but also intrinsically entangled to the availability of specific, low-coordinated sites, or the enhanced stability of reaction intermediates upon.

As already introduced the reagent adsorption has a key role in the catalytic process given that is a necessary step. Moreover, the location and adsorption strength of reactants may bias the posterior reaction steps. Nevertheless, bond breaking/forming steps are the essential steps in the course of a catalysed reaction, and, consequently, the posed methodology must be suited to explore such steps in order to get information correlated with the experimental observations. One of the first paramount works considering a catalytic process on metal nanoparticles was that of Yudanov et al., ${ }^{67}$ who studied the C-O bond dissociation on $\mathrm{Pd}$ nanocrystallites in the course of methanol decomposition. The authors investigated not only the adsorption of a handful of species on a cubo-octahedral $\mathrm{Pd}_{79}$ nanoparticle, including $\mathrm{CH}_{\mathrm{x}} \mathrm{OH}_{\mathrm{y}}(\mathrm{x}=0-3, \mathrm{y}=0-1)$, but also $\mathrm{CH}_{\mathrm{x}}(\mathrm{x}=0-3), \mathrm{OH}$, and $\mathrm{O}$ and $\mathrm{C}$ adatoms, but also the $\mathrm{C}-\mathrm{O}$ bond scission of the adsorbed $\mathrm{CH}_{\mathrm{x}} \mathrm{OH}_{\mathrm{y}}$ intermediates. The calculations, carried out with PARAGAUSS code, took profit of point-group symmetry, as already mentioned in various examples described in the previous section. Note, however, that in order to avoid artefacts and local restrictions, calculations were performed, when necessary, with symmetry reverted from $\mathrm{O}_{\mathrm{h}}$ to $\mathrm{D}_{4 \mathrm{~h}}$ or $\mathrm{D}_{4}$, i.e., revoking mirror planes. In this manner, the investigation of processes with adsorbates cross-edging neighbouring 
(111) facets is allowed. This restriction hinders the power of using point group symmetry, but infers versatility to the method. In any case, in order to alleviate the computational burden, ECP pseudopotentials were applied on the Pd core electrons. Calculations used also GTO basis sets and BP86 functional. Transition states were located using a constrained optimization approach, where reaction paths were explored by fixing the $\mathrm{C}-\mathrm{O}$ bond length and optimizing all other variables. The located transition states were characterized by a zero gradient and a Hessian matrix with only one negative eigenvalue.

Experimentally, it is found that methanol decomposition on Pd catalyst occurs with high selectivity toward $\mathrm{CO} / \mathrm{H}_{2}$. However, the overall activity is impeded by the catalyst modification by $\mathrm{CH}_{\mathrm{x}}$ deposits, mostly carbonaceous species. The DFT calculations of Yudanov et al. permitted to depict a rather clear picture of the methanol decomposition, see Figure 7, which provides an answer to the experimental observation, rendering a microscopic detail never gained before. Upon physisorption, the methanol molecule is prompted to lose one of the methyl hydrogen atoms. This dehydrogenation step was previously calculated to be exothermic by $45 \mathrm{~kJ} / \mathrm{mol}$ on a $\operatorname{Pd}(111)$ slab model $-V A S P$, PW91 - with a small activation barrier of $33 \mathrm{~kJ} / \mathrm{mol}$. On the nanoparticle the exothermicity of this step is found to be reduced to $25 \mathrm{~kJ} / \mathrm{mol}$. In subsequent steps the alcohol group of $\mathrm{CH}_{2} \mathrm{OH}$ intermediate is broken to form formaldehyde, $\mathrm{CH}_{2} \mathrm{O}$, and this latter molecule suffers consecutive dehydrogenations until forming CO. These last three steps were found to be exothermic by 19,61 , and $133 \mathrm{~kJ} / \mathrm{mol}$, respectively.

Concerning the carbon formation, it is unlikely to be originated from $\mathrm{CO}$ molecule dissociation in light of the calculations. This step is estimated to be highly endothermic $(241 \mathrm{~kJ} / \mathrm{mol})$, and features an even higher activation barrier of $414 \mathrm{~kJ} / \mathrm{mol}$. Therefore, the $\mathrm{C}$ formation is more sensible to occur via the breaking of the C-O bond from any of the $\mathrm{CH}_{\mathrm{x}} \mathrm{OH}_{\mathrm{y}}$ reaction intermediates. The calculations on the Pd nanoparticle models permitted to estimate that the lowest barriers for $\mathrm{C}-\mathrm{O}$ scission are found for $\mathrm{CH}_{3} \mathrm{O}$ and $\mathrm{CH}_{2} \mathrm{OH}$ intermediates, with activation barriers of 135 and $128 \mathrm{~kJ} / \mathrm{mol}$, respectively, i.e., few times lower than the barrier for $\mathrm{CO}$ molecule. Note also that the $\mathrm{C}-\mathrm{O}$ scission for $\mathrm{CH}_{3} \mathrm{O}$ is an exothermic process by $29 \mathrm{~kJ} / \mathrm{mol}$, yet $\mathrm{CH}_{2} \mathrm{OH} \mathrm{C}-\mathrm{O}$ scission is essentially isoenergetic (endothermic by $7 \mathrm{~kJ} / \mathrm{mol}$, within the method accuracy). Note that despite $\mathrm{CH}_{2} \mathrm{OH}$ is a reaction intermediate during $\mathrm{CO}$ formation, the $\mathrm{C}-\mathrm{O}$ scission form $\mathrm{CH}_{3} \mathrm{O}$ cannot be ruled 
out, since its formation from physisorbed methanol is endothermic by only $23 \mathrm{~kJ} / \mathrm{mol}$. In conclusion, the calculation on the $\mathrm{Pd}$ nanoparticle models led to the conclusion that carbonaceous formation is likely to proceed as a very slow by-process accompanying the dehydrogenation pathway; in particular, through the formation of $\mathrm{CH}_{3} \mathrm{O}$ or $\mathrm{CH}_{2} \mathrm{OH}$ intermediates and eventual C-O bond scission. Yudanov et al. also found that once $\mathrm{CH}_{3}$ and $\mathrm{CH}_{2}$ species are formed, the following dehydrogenation steps are estimated to be exothermic by 11 and $37 \mathrm{~kJ} / \mathrm{mol}$, respectively, whereas $\mathrm{C}$ adatom formation from $\mathrm{CH}$ is moderately endothermic $(29 \mathrm{~kJ} / \mathrm{mol})$. All in all, the formation of carbonaceous species during the methanol decomposition into $\mathrm{CO} / \mathrm{H}_{2}$ seems to be determined firstly by an easier C-O bond scission on $\mathrm{CH}_{3} \mathrm{O}$ and $\mathrm{CH}_{2} \mathrm{OH}$ intermediates, biased by the single ( $\sigma$-type) $\mathrm{C}-\mathrm{O}$ bond breaking, in juxtaposition to $\mathrm{C}-\mathrm{O}$ scission from $\mathrm{CO}$ molecule or other intermediates, which resulted in larger activation energy barriers due strengthening $\pi$-contribution to the $\mathrm{C}-\mathrm{O}$ bond. Besides, the process is also equally influenced by the preferential occupation of $\mathrm{CH}_{3}$ and $\mathrm{CH}_{2}$ species at the edges of the Pd nanoparticles, whose binding energies are 65 and $36 \mathrm{~kJ} / \mathrm{mol}$ stronger than on the middle of the (111) facet, inherently prompting the $\mathrm{C}-\mathrm{O}$ bond scission at these sites.

It is worth to mention that a subsequent DFT theoretical work combining $\operatorname{Pd}(111)$ slab and $\mathrm{Pd}_{140}$ nanoparticle models showed that $\mathrm{C}$ adatoms are thermodynamically driven to occupy octahedral or tetrahedral subsurface sites. ${ }^{68}$ On the $\operatorname{Pd}(111)$ surface, subsurface diffusion of $\mathrm{C}$ adatoms features distinctive activation energy barriers ranging $50-60 \mathrm{~kJ} / \mathrm{mol}$, where subsurface octahedral sites are clearly preferred over subsurface tetrahedral sites. In contrast, on the $\operatorname{Pd}_{140}$ nanoparticle low-coordinated sites, such as edges and corners, it is observed a sensible stabilization of subsurface C, by up to $\sim 30 \mathrm{~kJ} / \mathrm{mol}$, when occupying subsurface tetrahedral sites; thus levelling its stability to vicinal octahedral sites. Moreover, while the subsurface diffusion energy barrier for $\mathrm{C}$ adatoms located at the centre of $\operatorname{Pd}_{140}$ (111) facets is similar to that obtained on $\operatorname{Pd}(111)$ slab, the barriers drop to a few $\mathrm{kJ} / \mathrm{mol}$ or essentially vanish for sites nearby or located at edges and corners. The key role of lowcoordinated sites in having subsurface $\mathrm{C}$ species was found to be intimately correlated with the enhanced fluxionality of metal atoms at these sites, which is not only allowing for a better accommodation of species upon or within, but here also at the origin of the substantial subsurface diffusion activation energy reduction. 
We now come back to the particular stabilization of $\mathrm{CH}_{3}$ and $\mathrm{CH}_{2}$ species at unsaturated sites, which was also detected in the study of Viñes et al. concerning the methane activation by $\mathrm{Pt}$ nanoparticles. ${ }^{58}$ Here, $\mathrm{CH}_{4}$ adsorption and its complete dehydrogenation was studied on a $\mathrm{Pt}_{79}$ nanoparticle at $\mathrm{RPBE}$ level. In contrast to the previous study by Yudanov et al., ${ }^{67}$ who used a molecular code, the authors carried out the calculations with the VASP code, in which periodic conditions are imposed. This choice enabled one to get rid of point group symmetry constraints and to study the decomposition of a single molecule per particle, but also allowed direct comparison with extended slab calculations using the same basis set and calculation parameters. That was indeed the case for methane dissociation, where a split-and-win modelling strategy was applied to model Pd nanoparticles of a few $\mathrm{nm}$ in size, in which slab calculations served to model inner parts of facets, whereas the $\mathrm{Pt}_{79}$ nanoparticle permitted to simulate the catalytic activity of lowcoordinated sites, such as edges and corners. Dehydrogenation steps activation energy barriers were obtained by locating the transition states using the climbing image nudged elastic band (CI-NEB), and, as done in the work of Yudanov et al., ${ }^{67}$ they were characterized as so by a vibrational frequency analysis, a fact that allowed to correct the energy profiles with the zero point energy (ZPE) vibrations.

As already introduced, methane dehydrogenation $\mathrm{CH}_{3}$ and $\mathrm{CH}_{2}$ intermediate species were found to be stabilized, by $50-80 \mathrm{~kJ} / \mathrm{mol}$, at particle edges and corners. For other reaction species such stabilization also occurs, but to a lesser extent. This enhanced activity seems to stem out from two factors: low-coordinated sites are intrinsically more active to bind species upon due to their unsaturation. Besides, as found for the subsurface $\mathrm{C}$ on $\mathrm{Pd}$ nanoparticles, ${ }^{68}$ the metal atoms at these sites feature a natural larger flexibility to accommodate adsorbates. This, a priori, simple factors can be translated into substantial differences in both the thermodynamics and kinetics of the reaction. See, for instance, the reaction profiles displayed in Figure 8 for the $\mathrm{CH}_{4}$ complete dehydrogenation on the $\mathrm{Pt}(111)$ surface and on the $\mathrm{Pt}_{79}$ nanoparticle. One of the main points is that methyl and methylene formation reaction steps are endothermic for single crystal surfaces, whereas they are clearly exothermic on $\mathrm{Pt}_{79}$. This has a remarkable effect on the first methane $\mathrm{C}-\mathrm{H}$ bond scission, which is normally the rate-limiting step due to the high $\mathrm{CH}_{4}$ stability. The methane dehydrogenation barrier is $\sim 60 \mathrm{~kJ} / \mathrm{mol}$ reduced compared to the one on $\operatorname{Pt}(111)$ 
surface. Furthermore, a similar reduction of $\sim 40 \mathrm{~kJ} / \mathrm{mol}$ is observed for methyl decomposition. Regardless of the substrate, or, in other words, the catalyst site, $\mathrm{CH}$ species seems to be the most likely end of the reaction. However, $\mathrm{C}$ adatom formation is found to be thermodynamically driven at particle edges and corners, although overcoming a high energy barrier of almost $100 \mathrm{~kJ} / \mathrm{mol}$. The computationally derived scenario fits perfectly with the single crystal and nanoparticle experiments. On single crystal surfaces, methyl species are found to be major species when dosing methane with a supersonic beam. Only after heating recombination of methyl species is observed, with the simultaneous desorption of $\mathrm{CH}_{4}$ and formation of $\mathrm{CH}$ and $\mathrm{C}$ species. On nanoparticles, the X-ray photoelectron spectroscopy (XPS) measurements reveal a rapid formation of methyl species (at facets and edges), followed by the sequential formation of $\mathrm{CH}$ and $\mathrm{C}$ species (at edges and corner sites) at surface temperatures as low as $100 \mathrm{~K}$, in concordance with the reduced energy barrier for methyl decompositions, as observed in Figure $8 .^{58}$

One interesting point of this study is that the exothermicity increase of a reaction step — originated by an enhanced stability of the formed species - is accompanied by a reduction of the reaction step activation energy barriers. In this regard Brønsted-EvansPolanyi (BEP) relationships linearly relating the activation energy for a given elementary step with its corresponding reaction energy can be summoned. This aspect was succinctly introduced in the work by Viñes et al., ${ }^{58}$ but fully investigated in a recent work by Fajín and co-workers on the water dissociation on Pt nanoparticles, ${ }^{65}$ studied as an elementary step of the steam reforming of methanol or methane carried out on Pt model catalysts. Fajín et al. studied the adsorption of a single water molecule and its homolytic dissociation on $\mathrm{Pt}_{13}$, $\mathrm{Pt}_{19}, \mathrm{Pt}_{38}, \mathrm{Pt}_{55}, \mathrm{Pt}_{79}$, and $\mathrm{Pt}_{140}$ nanoparticles, and compared the process to the extended $\operatorname{Pt}(111)$ slab situation, carrying out DFT calculations with the PW91 exchange-correlation functional. Similarly to the above-commented methane dehydrogenation study, authors took profit of a periodic code, found transition states with the Dimer method, and identified them as so with a vibration analysis. Reaction profiles were ZPE-corrected. As a step further, reaction rate constant estimations were acquired from transition state theory, thus fully exploiting the theoretical machinery in the description of a catalysed reaction. In that work, the effect of spin-polarization was explicitly addressed, since it was found for small $\mathrm{Pt}$ clusters, such as $\mathrm{Pt}_{8}$, that the optimal number of unpaired electrons had a direct impact 
on the cluster activity. However, for larger nanoparticles either closed shell states were found or the spin-polarized solution was only minimally lower in energy, $\sim 0.1 \mathrm{~kJ} / \mathrm{mol}$, with negligible effect on the cluster or nanoparticle catalytic activity. As commented in the previous section, the usage of smaller clusters or larger nanoparticles leaded to small changes in the adsorption of $\mathrm{H}_{2} \mathrm{O}$, presumably due to the weak sorption of water (physisorbed state), which makes it structure/substrate-independent. This is not the case, though, for the estimated $\mathrm{H}_{2} \mathrm{O}$ dissociation energy barriers: as it is shown in Figure 9, the activation energy barriers sensibly oscillate for the smaller clusters; a clear reflection that such nanoparticles are out of the scalable regime. However, $\mathrm{Pt}_{140}$ barrier is of the same order of the $\operatorname{Pt}(111)$ limit, indicating that convergence with size is reached. In addition authors found linear dependency of the activation energies with respect to the degree of exothermicity, i.e., a BEP relationship, although the fitting was less quantitative than previous calculations exploring water splitting on the (111) surface of different transition metals. One particularly interesting point of such study is that $\mathrm{H}_{2} \mathrm{O}$ dissociation is likely to occur since the dissociated species are energetically equal or lower than adsorbed water, but also it is a requirement that the dissociation transition state lies lower in energy than the desorbed water situation. In other words, if the transition state corresponding to the reaction of $\mathrm{H}_{2} \mathrm{O}$ dissociation lies above the energy level of a non-interacting system containing the water molecule and the Pt nanoparticle, then the adsorbed molecule is likely to desorb rather than to decompose. Having this in mind, the authors found that the larger the nanoparticle, the less favourable the water dissociation is: optimum size is found for (sub)nanometer size clusters.

The particular aspect above was the leitmotiv in the investigations of Roldán et al. concerning the $\mathrm{O}_{2}$ dissociation on Au nanoparticles, ${ }^{69,70}$ a work triggered by the effective catalysis of $\mathrm{Au}_{55}$ on the styrene selective oxidation, ${ }^{39}$ or the low temperature oxidation of $\mathrm{CO}$, as found by Haruta when supporting $\mathrm{Au}$ on $\mathrm{TiO}_{2}{ }^{71}$ Authors explored the catalytic activity of a series of $\mathrm{Au}_{\mathrm{n}}$ clusters $(\mathrm{n}=5,13,25,38,55,79,116$, and 147) by studying the $\mathrm{O}_{2}$ adsorption and dissociation upon, using the VASP code and the PW91 exchangecorrelation functional. Transition states were located by combining CI-NEB and Dimer methods, and they were confirmed as transitions states via a vibrational analysis. One of the most striking findings was to unravel that the $\mathrm{O}_{2}$ dissociation activity was probably not due 
to the $\mathrm{Au}_{55}$ nanoparticle, as previously suggested. For this particularly stable nanoparticle, $\mathrm{O}_{2}$ desorption required significantly less energy $(18 \mathrm{~kJ} / \mathrm{mol})$ than dissociation (energy barrier of $71 \mathrm{~kJ} / \mathrm{mol}$ ). The same situation was noticed for larger clusters, with adsorption energies ranging 30-50 kJ/mol, and $\mathrm{O}_{2}$ dissociation activation energies ranging 40-55 $\mathrm{kJ} / \mathrm{mol}$. Thus, energy barriers only slightly depend on the particle size, whereas a larger variation is found for adsorption energies. This was observed to be intimately related with the existence of a gap in between the occupied and unoccupied states of the pristine nanoparticle: when a gap exists, like in $\mathrm{Au}_{38}$, it allows accommodating bonding states with $\mathrm{O}_{2}$ below the Fermi level, resulting in a stronger interaction, while in the absence of a gap, i.e., metallic electronic structure, as happens for larger $\mathrm{Au}$ nanoparticles, the interaction renders bonding and antibonding states, resulting in a weaker $\mathrm{O}_{2}$ interaction. Thus, $\mathrm{O}_{2}$ dissociation seems to be strongly governed by the $\mathrm{O}_{2}$ adsorption strength, in contrast to $\mathrm{H}_{2} \mathrm{O}$ dissociation on $\mathrm{Pt}$ nanoparticles, where the activation energy barrier was governing the process. In fact, it is for $\mathrm{Au}_{38}$ particle that authors found an adsorption energy $(88 \mathrm{~kJ} / \mathrm{mol})$ larger than the dissociation energy barrier $(44 \mathrm{~kJ} / \mathrm{mol})$, suggesting that this -and other clusters of similar size- are well-suited for the $\mathrm{O}_{2}$ dissociation catalysis. Thus, the experimental observation of the high activity of $\mathrm{Au}_{55}$ in $\mathrm{O}_{2}$ dissociation is maybe apparent, and the activity is due to smaller clusters, given that in the experiments a distribution of $\mathrm{Au}$ nanoparticles was obtained. Note that this situation however can diverge for very small clusters: for instance, the $\mathrm{O}_{2}$ desorption and dissociation are both competitive situations on $\mathrm{Au}_{25}$, whereas for smaller clusters, $\mathrm{Au}_{5}$ and $\mathrm{Au}_{13}$, the dissociation barriers are markedly higher, 271 and $279 \mathrm{~kJ} / \mathrm{mol}$, respectively, although adsorption energy is still calculated to be $\sim 50 \mathrm{~kJ} / \mathrm{mol}$. Moreover, the $\mathrm{O}_{2}$ dissociation paths are found to be intimately ligated to the existence of (001) like facets, especially on the medium and large size nanoparticles, highlighting the key role of the existence of such facets in the $\mathrm{O}_{2}$ dissociation and, presumably, on the commented Au-catalysed reactions, a fact associated to the specific nanoparticle shape.

In order to mimic possible charge transfer effects arising from the presence of a support, the same authors investigated the effect on negative and positive charge on such $\mathrm{Au}$ nanoparticles, i.e., considered the charge effect on $\mathrm{O}_{2}$ adsorption and dissociation. This is a simple but efficient way to emulate the possible slight reduction/oxidation of such gold 
nanoparticles when supported onto an oxide substrate. ${ }^{72}$ Beyond the non-scalability region found for clusters below $\sim 80$ atoms, already commented, the study unfolded that the addition of positive/negative charge has a significant effect on ultra-small clusters containing $<20$ atoms. In principle, the negative charge is beneficial in the sense that lowers the $\mathrm{O}_{2}$ dissociation energy barrier and stabilizes the final products. However, as mentioned, the barriers are quite high for such clusters, and the $\mathrm{O}_{2}$ dissociation is unlikely because the molecule would rather desorb. The opposite trend is found when adding a positive charge to the Au nanoparticle, i.e., removing one electron. Indeed such trends are found for any of the studied sizes, although the effect is rather mild for medium $\left(\mathrm{Au}_{38}\right.$ and $\left.\mathrm{Au}_{55}\right)$ and large nanoparticles $\left(\mathrm{Au}_{79}\right)$. Curiously, the inclusion of charge does not ameliorate the catalytic activity on $\mathrm{O}_{2}$ dissociation, this is, $\mathrm{Au}_{38}$ is active and the other particles are not, regardless of the charge state. Last but not least it was found that adsorption geometry, binding strength, barrier height, and products stability are mostly independent of the cluster charge state.

These previous studies triggered a theoretical work by Boronat and Corma, where $\mathrm{O}_{2}$ dissociation was investigated on $\mathrm{Au}(001)$ surface, given the key role of such sites on the process, on $\mathrm{Au}_{38}$ nanoparticle, given the suited catalytic activity reported, and on a hemispherical $\mathrm{Au}_{13}$ cluster unsupported or supported on a $\mathrm{TiO}_{2}$ slab, in both cases containing the same active site as in the $\mathrm{Au}_{38}$ nanoparticle. DFT calculations were carried out using VASP code and the PW91 exchange-correlation functional; transition states were obtained using the Dimer method, and characterized by the pertinent frequency analysis calculations. The results showed that $\mathrm{O}_{2}$ dissociation on regular $\mathrm{Au}(001)$ surface is not viable, given that the activation barrier of $42 \mathrm{~kJ} / \mathrm{mol}$ is larger than the $\mathrm{O}_{2}$ desorption energy of $21 \mathrm{~kJ} / \mathrm{mol}$. Results on $\mathrm{Au}_{38}$ confirmed the previous findings of Roldán et al., ${ }^{69}$ whereas for unsupported $\mathrm{Au}_{13}$ the dissociation path in which $\mathrm{O}_{2}$ is attaching with a binding strength of $95 \mathrm{~kJ} / \mathrm{mol}$ to a bridge-bridge site at (001) facet leads to a dissociation through a small energy barrier of $17 \mathrm{~kJ} / \mathrm{mol}$. In fact, the highest degree of molecular activation is always related to the existence of bridge-bridge conformers on (001) surfaces or facets. Additionally, the authors found, nevertheless, that the easiest path to dissociate $\mathrm{O}_{2}$ on $\mathrm{Au}_{13}$ is when supported on $\mathrm{TiO}_{2}$. In the latter, $\mathrm{O}_{2}$ is preferentially adsorbed at the metal-support interface, i.e., at the boundary region of $\mathrm{Au}_{13}$ cluster, thus in close contact to both the gold 
cluster and the titania substrate. The adsorption energy of $232 \mathrm{~kJ} / \mathrm{mol}$ is accompanied by a moderately high dissociation energy barrier of $106 \mathrm{~kJ} / \mathrm{mol}$, highlighting the possible lowrate side process at boundary regions. In any case, such study must be taken with caution since it deals with a cluster out of the scalable regime, and thus the results should not be extrapolated to larger nanoparticles, since this peculiar activity could be solely attributed to this particular particle. In any case the results tend to suggest a rapid $\mathrm{O}_{2}$ dissociation on (001) facets of small Au clusters, and a slow by-process at the metal support interface.

\section{Concluding remarks and perspectives}

The previous sections have illustrated how suitable models of metallic nanoparticles can be build and how these models can be used to extract general trends about their adsorption properties and their chemical reactivity towards several simple but important reactions. The studies presented are not the only ones in the literature but they have been chosen because are representative enough, because involve nanoparticles of different size allowing one to derive trends and, finally, because constitute clear examples where the potential energy surface has been explored and transition state structure determined. Clearly, for very large nanoparticles, surface sites can be represented by suitable periodic slab models. This is not only the case for terrace sites but also for edge sites at the intercept of surfaces with different Miller indexes which can be featured by stepped surface slab models too although the size of the unit cell becomes large. However, these models, while valid and useful, miss the particularities that size and shape confer to the nanoparticles mostly described in the present work and which allowed to derive a few general rules.

The systems reviewed in the present work have been deliberatively chosen as monometallic, all atoms in the nanoparticle are of the same chemical element. Bimetallic particles constitute yet another type of interesting systems for which abundant literature exists either from theory or experiment which is not reviewed here although we must mention that composition and order of elements can give to core-shell or amorphous particles each with its own particularities in structure and chemical reactivity. The atomic structure of isolated bimetallic nanoparticles is a hot field nowadays although theoretical studies concerning the reactivity of these systems are scarce.

Last but not least one must realize that metallic nanoparticles as presented in this work are ideal entities since these objects are usually found either covered by stabilizing 
agents or deposited on convenient supports. The reactivity of supported nanoparticles is also a field in itself although quite in its infancy. Again, for very large particles the effect of the support can be neglected and the chemistry is dominated by the exhibited surface sites. However, for small or medium particles the support can play an active role an largely influence the chemistry. It is precisely in this type of systems where engineering at the molecular level it likely to be possible and to play a crucial role. The type of systems described at the bottom of the previous systems are clear examples that this chemistry is not only at hand but can be understood to design more active and efficient catalysts.

\section{Acknowledgments}

This work was supported by the Spanish MICINN and MINECO (FIS2008-02238 and CTQ2012-30751 grants, respectively) and by Generalitat de Catalunya (grants 2009SGR1041 and XRQTC). F.V. thanks the MINECO for a postdoctoral Juan de la Cierva grant (JCI-2010-06372), F.I. acknowledges additional support through the ICREA Academia award for excellence in research and JRBG thanks Fundação para a Ciência e a Tecnologia (FCT), Lisbon, and European Regional Development Fund (FEDER), within the frame of the COMPETE program for projects PTDC/QUI-QUI/117439/2010, FCOMP01-0124-FEDER-020977, and PEst-C/CTM/LA0011/2013, and for Programa Investigador FCT. 


\section{Caption for Figures}

Figure 1.- Schematic representation of (a) a $\mathrm{Pt}_{8}$ gas phase cluster, (b) a three layer $\mathrm{Pt}_{29}$ cluster model representation of $\mathrm{Pt}(100)$, and (c) a $\mathrm{Pt}_{598}$ cubooctahedral nanoparticle exhibiting (111) and (100) facets.

Figure 2.- Schematic representation of the Wulff construction for (a) a two dimensional case and (b) for a three dimensional particle.

Figure 3.- Variation of an arbitrary property with the nanoparticle size either towards bulk (top panel) or towards the extended surface limit (bottom panel), illustrating scalable and the non-scalable regimes.

Figure 4.- Variation of the average nearest distances $d(\mathrm{Pd}-\mathrm{Pd})$ of $\mathrm{Pd}$ nanoparticles with the average coordination number $N_{\mathrm{av}}$. The solid line correspond to the plane-wave calculations of Viñes et al. ${ }^{56}$ whereas dashed line correspomnd to the all electron scalar relativistic cluster calculations of Yudanov et. al. ${ }^{42}$ Adapted with permission from F. Viñes, F. Illas, K.M. Neyman, J. Phys. Chem. A 2008, 112, 8911-8915. Copyright (2008) American Chemical Society.

Figure 5.- Views of metal particles with $\mathrm{O}_{\mathrm{h}}$ symmetry with nuclearities between 55 and 148.

Figure 6.- Trends in the calculated adsorption energies $\left(\mathrm{E}_{\mathrm{ads}}\right)$ for $\mathrm{CO}$ adsorption on $\mathrm{Pd}_{\mathrm{n}}$ particles as a function of the effective particle diameter $\left(D_{\text {eff }}\right)$. The yellow rectangle marks the critical size range, i.e., particles with $\sim 50 \mathrm{Pd}$ atoms, where the dominant interaction mechanism changes from that associated with the non-scalable regime (full line, $\mathrm{E}_{\mathrm{ads}}$ decreases with $\mathrm{D}_{\text {eff }}$ ) to the scalable regime (dot-dashed line, $\mathrm{E}_{\mathrm{ads}}$ increases with $\mathrm{D}_{\text {eff }}$ ). The dotted line represents the extrapolated $\mathrm{E}_{\mathrm{ads}}$ for $\mathrm{CO}$ adsorption on an infinite $\operatorname{Pd}(111)$ surface. Adapted from ref. 60. 
Figure 7.- Methanol and selected intermediates of its decomposition: reaction energies ( $\mathrm{kJ} / \mathrm{mol}$ ) for the splitting of $\mathrm{CH}, \mathrm{OH}$, and $\mathrm{CO}$ bonds as well as for $\mathrm{CH}_{3} \mathrm{OH}, \mathrm{CH}_{2} \mathrm{O}$, and $\mathrm{CO}$ desorption as calculated on (111) facets of $\mathrm{Pd}_{79}$. Main reaction path is highlighted by green colours, whereas $\mathrm{CO}$ dissociation barriers are denoted by orange lines and numbers. Adapted with permission from I. V. Yudanov, A. V. Matveev, K. M. Neyman, and N. Rösch, J. Am. Chem. Soc. 2008, 130, 9342-9352. Copyright (2008) American Chemical Society.

Figure 8.- ZPE-corrected reaction energy profile for the complete dehydrogenation of methane on a $\mathrm{Pt}(111)$ surface and on a $\mathrm{Pt}_{79}$ nanoparticle. All energies, in $\mathrm{kJ} / \mathrm{mol}$, refer to methane in the gas phase and the clean substrate. In the sketches of the transition states, only small portions containing seven Pt atoms of the calculated slab and cluster models are displayed for the sake of visual recognition. Adapted with permission from F. Viñes, Y. Lykhach, T. Staudt, M. P. A. Lorenz, C. Papp, H.-P. Steinrück, J. Libuda, K. M. Neyman, and A. Görling, Chem. Eur. J. 2010, 16, 6530-6539. Copyright (2010) Wiley-VCH Verlag $\mathrm{GmbH} \& \mathrm{Co}$.

Figure 9.- Activation energy barrier with respect to the number of atoms in the $\mathrm{Pt}$ nanoparticles or extended (111) surface. Adapted with permission from J. L. C. Fajín, A. Bruix, M. N. D. S. Cordeiro, J. R. B. Gomes, and F. Illas J. Chem. Phys. 2012, 137, 034701. Copyright (2012) American Institute of Physics. 
Figure 1.-

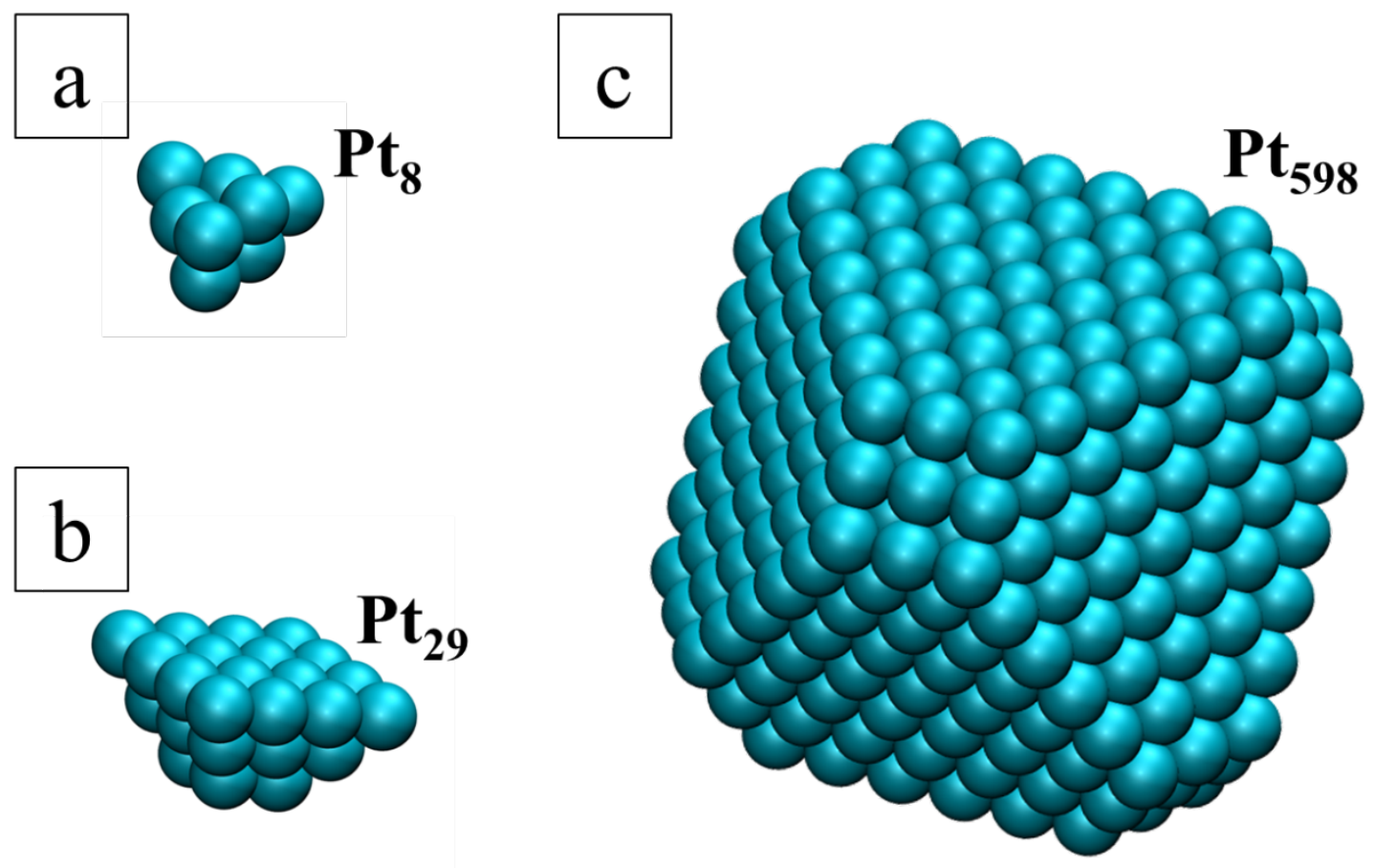


Figure 2
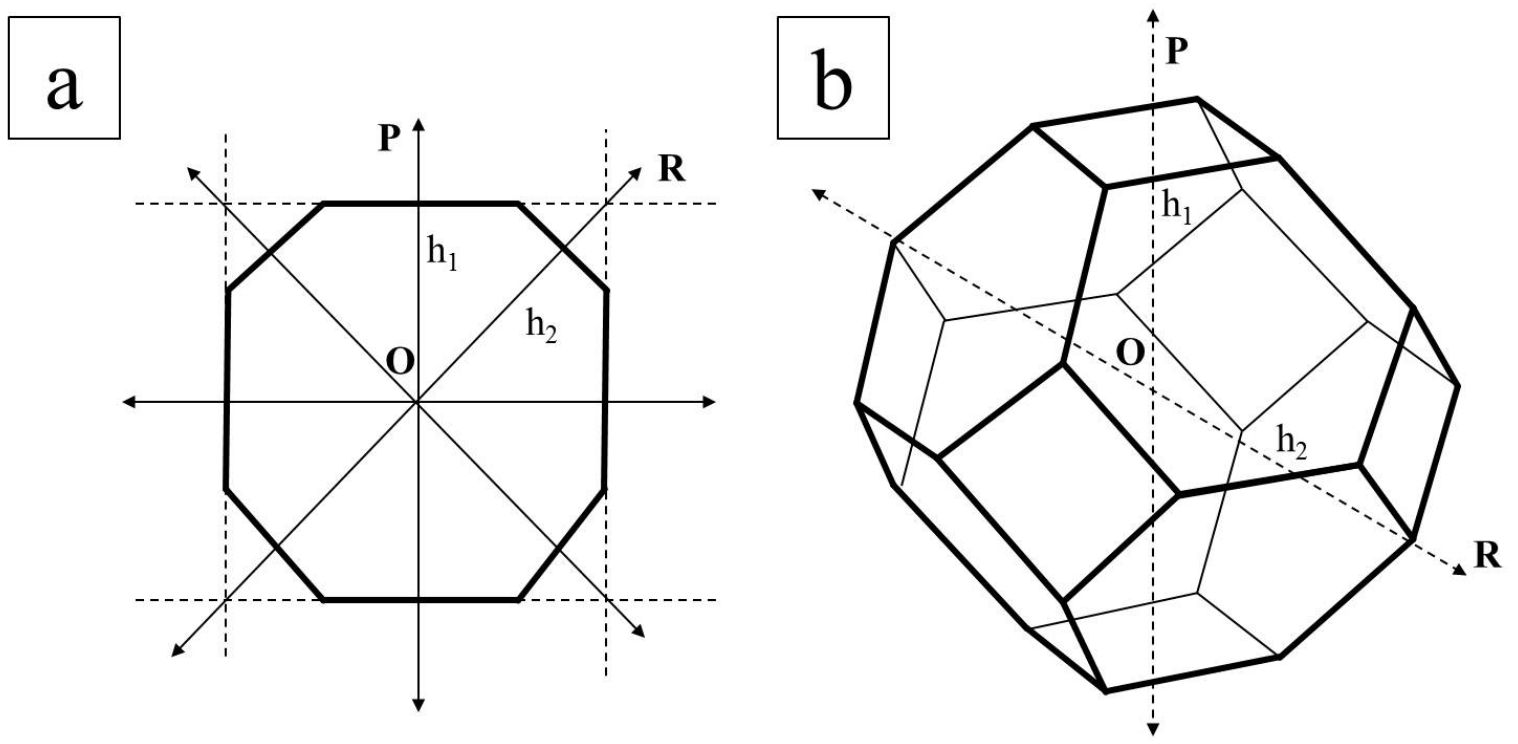
Figure 3

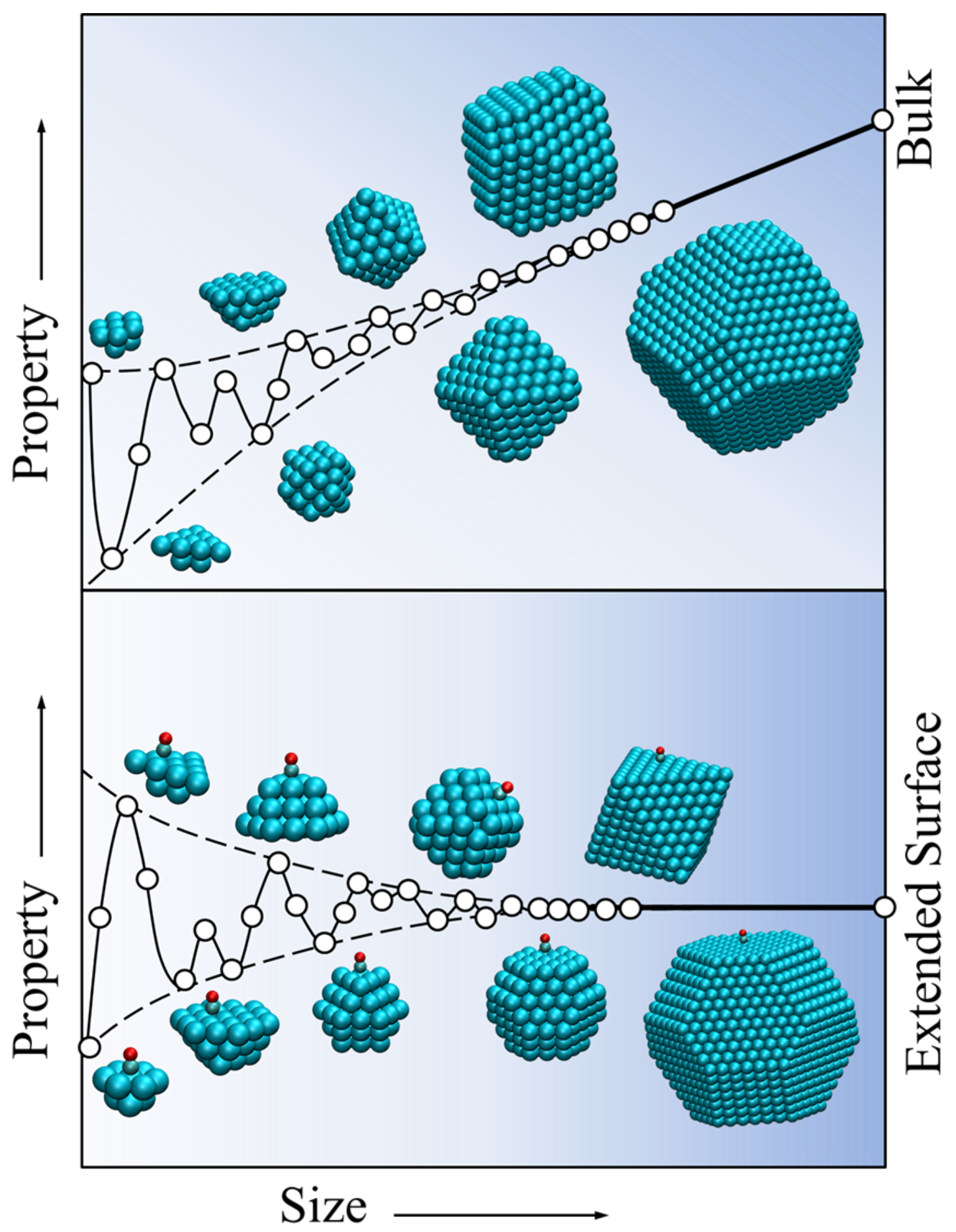


Figure 4.

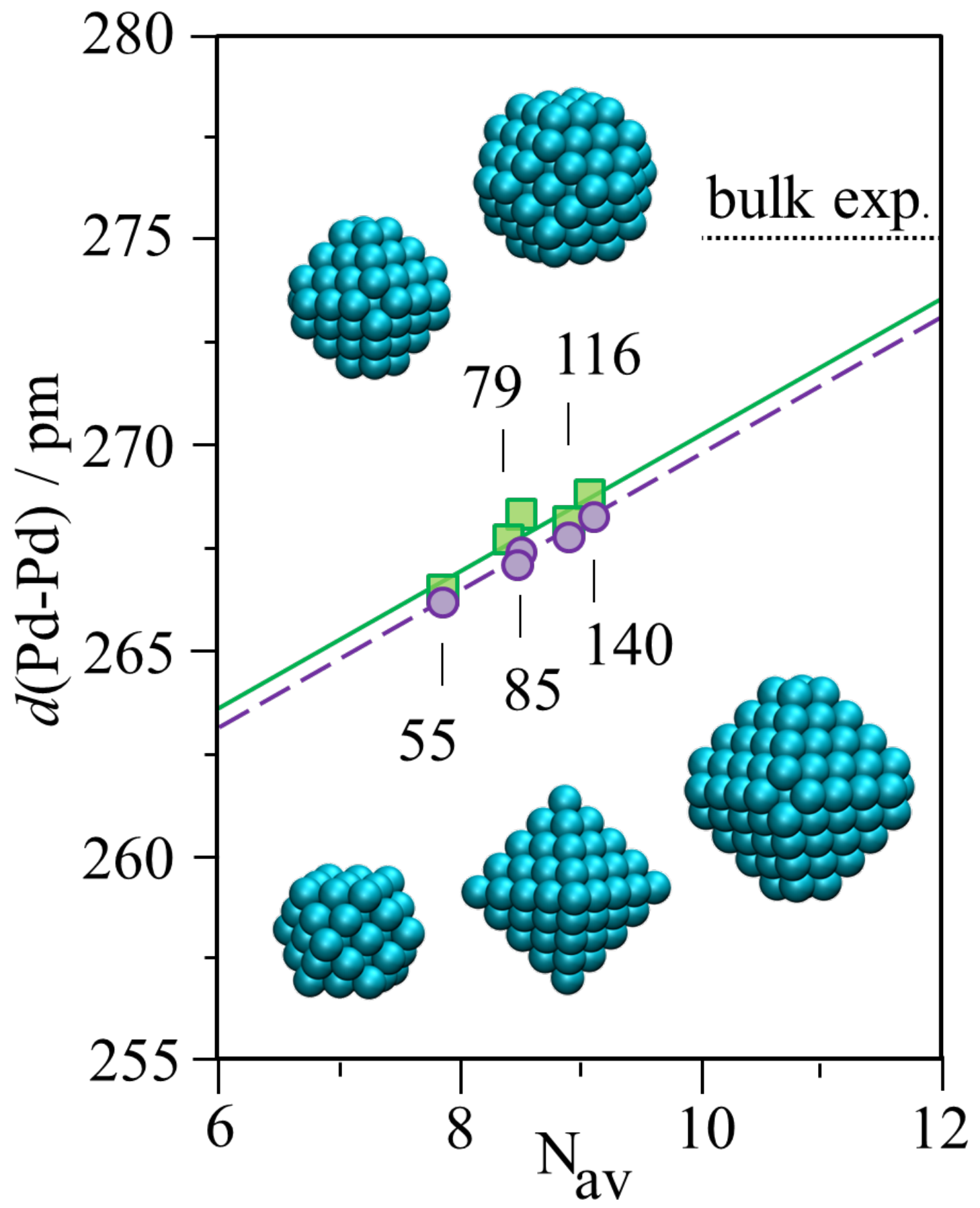


Figure 5.

55

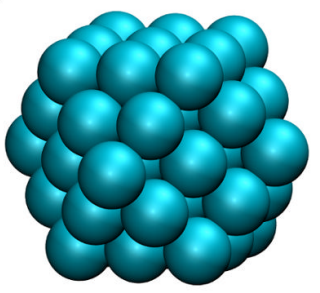

79

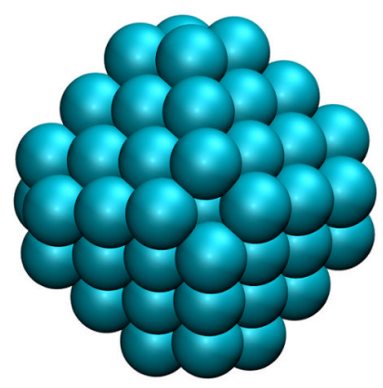

85

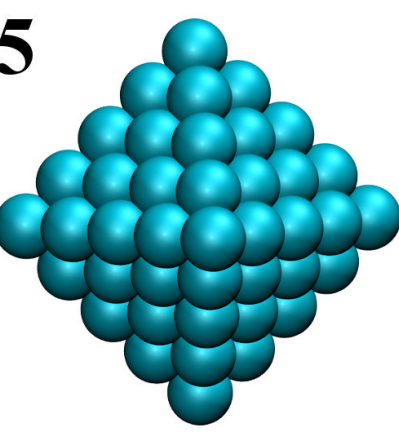

116

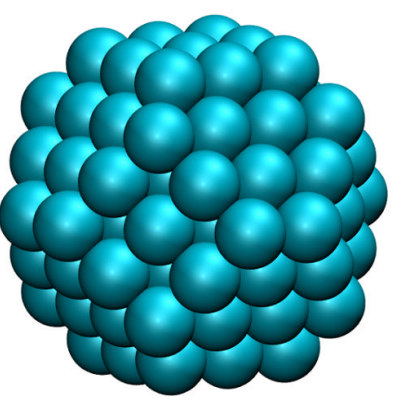

140
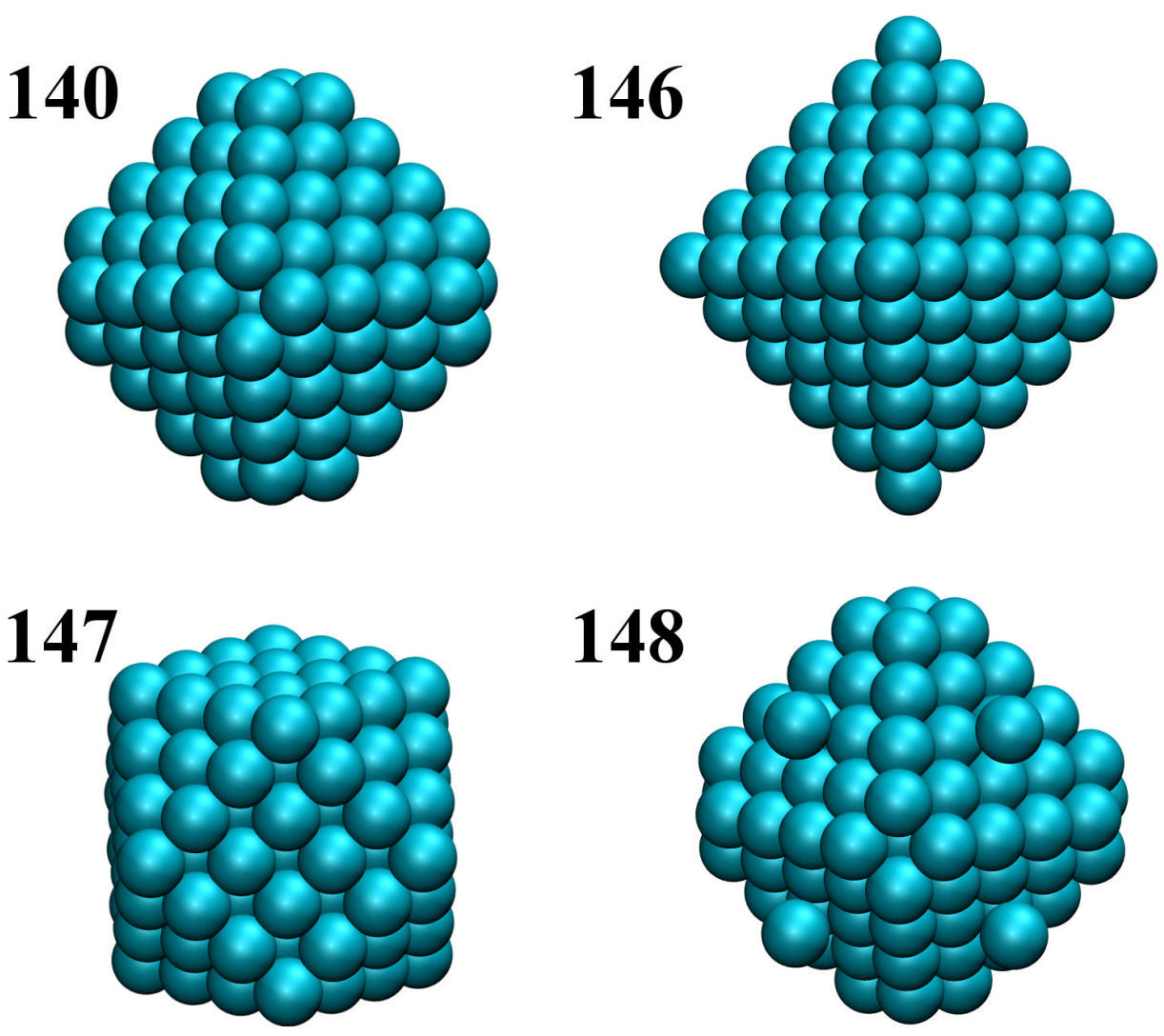
Figure 6.

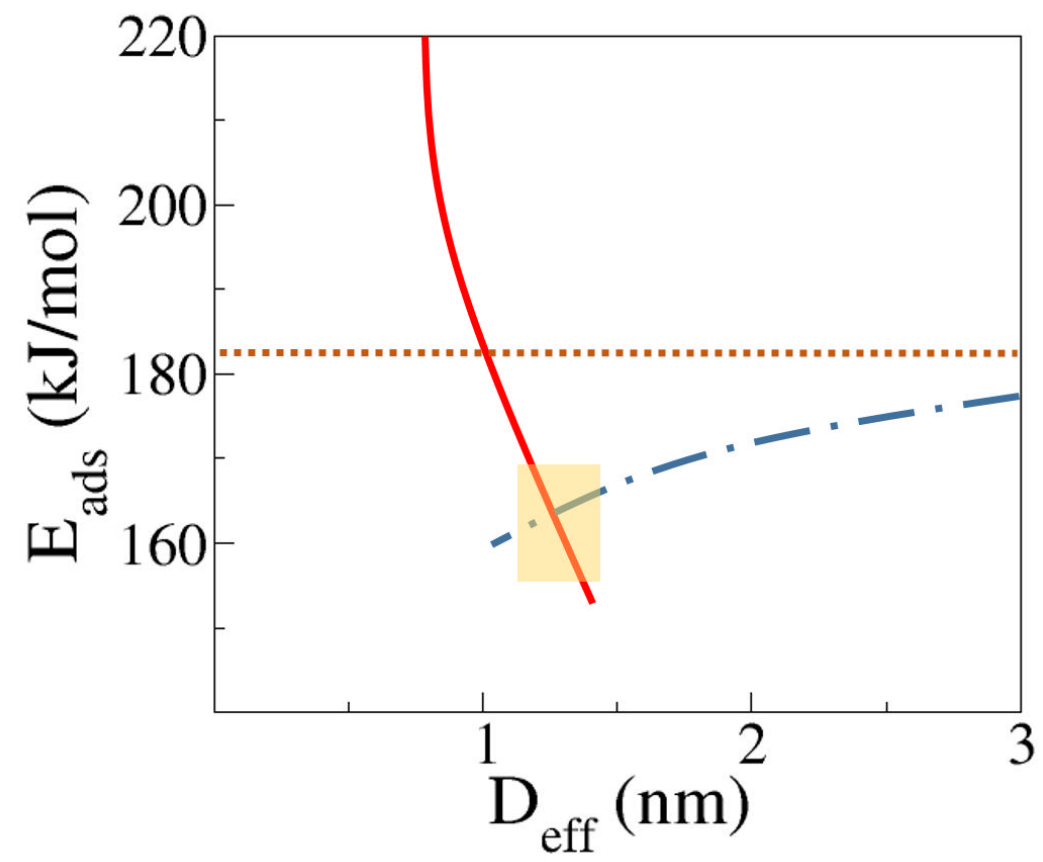


Figure 7.

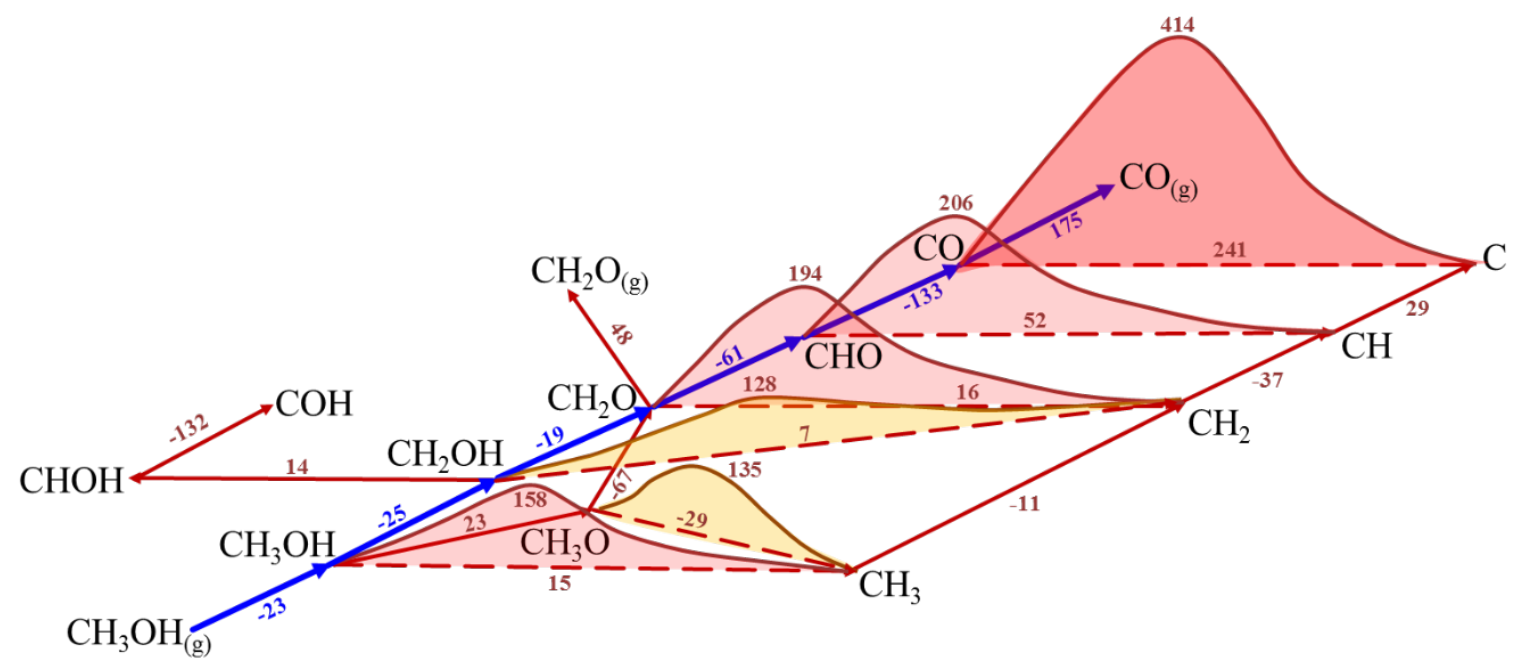


Figure 8.

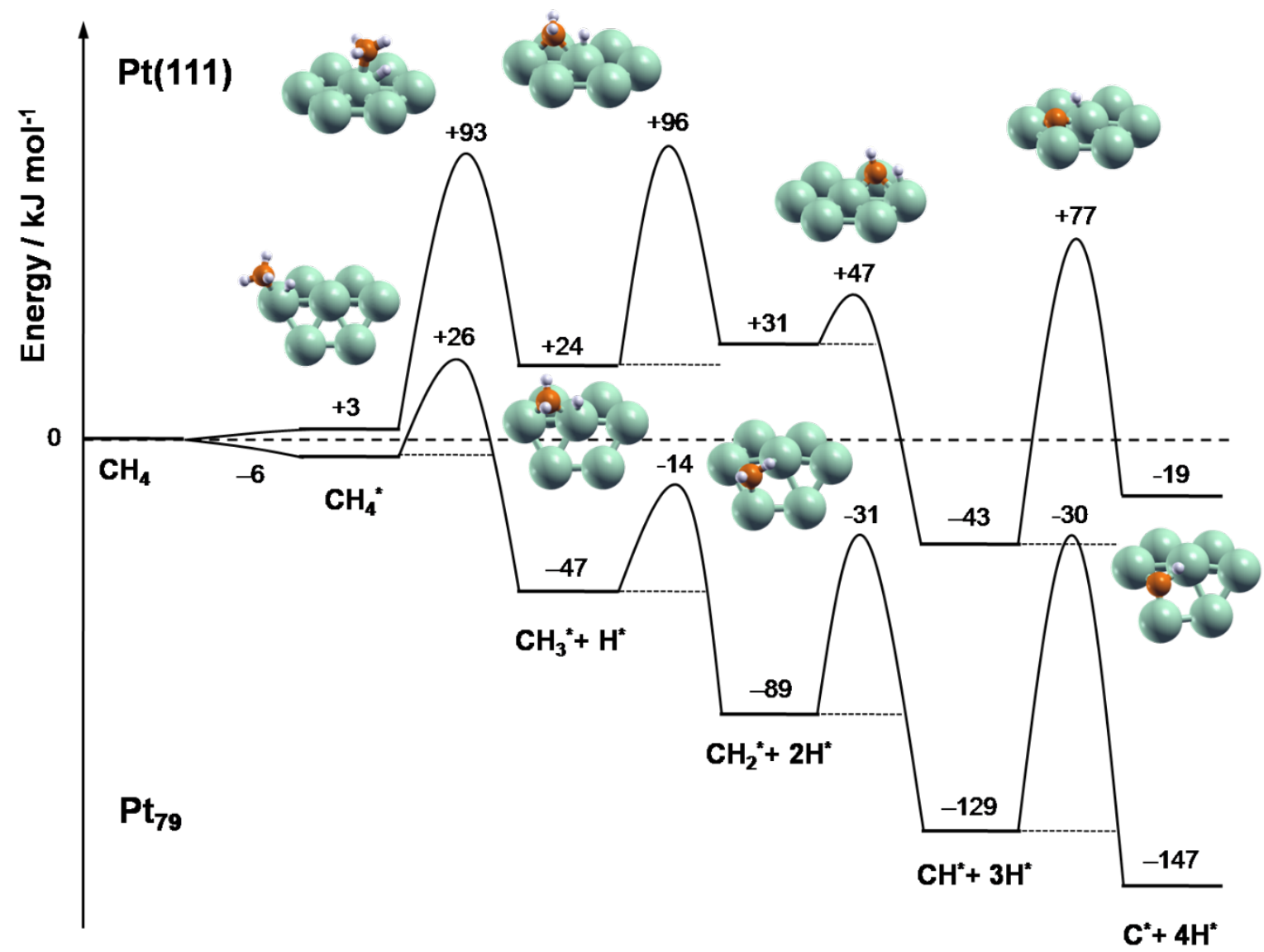


Figure 9.

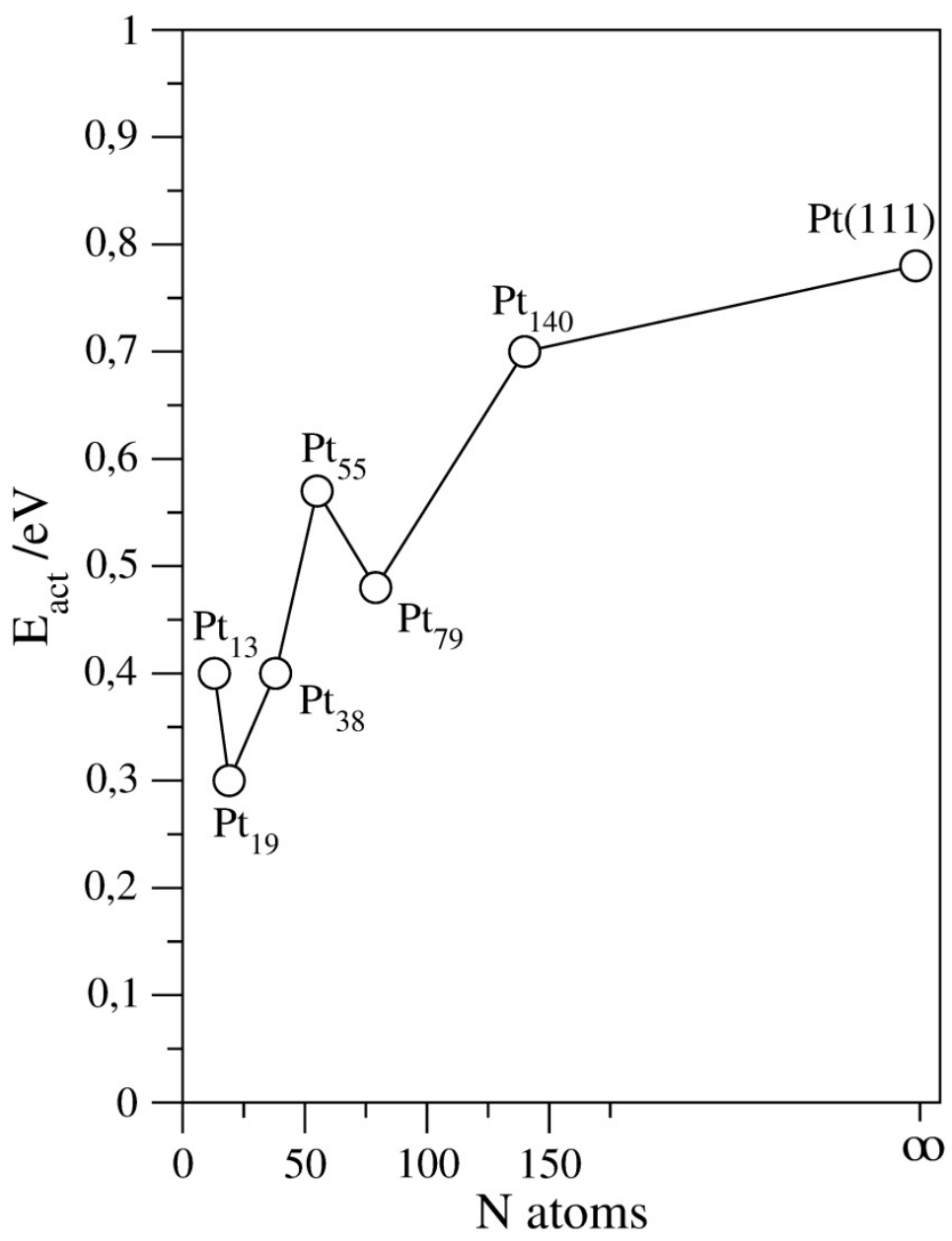




\section{References}

$1 \quad$ M. A. El-Sayed, Acc. Chem. Res., 2001, 34, 257-264.

2 K. L. Kelly, E. Coronado, L. L. Zhao, G. C. Schatz, J. Phys. Chem. B, 2003, 107, 668677.

3 B. C. Gates, Chem. Rev., 1995, 95, 511-522.

4 G. Ertl, Handbook of Heterogeneous Catalysis, Wiley-VCH, Weinheim, 2008.

5 Supported Metals in Catalysis, $2^{\text {nd }}$ Edition, J. A. Anderson and M. Fernández-García Eds., Catalytic Science Series: Volume 11, Imperial College Press, London, 2011.

6 P. V. Kamat, J. Phys. Chem. B, 2002, 106, 7729-7744.

7 M. T. Reetz, W. Helbig, J. Am. Chem. Soc., 1994, 116, 7401-7402.

8 Y. G. Sun, Y. N. Xia, Science, 2002, 298, 2176-2179.

9 Y. N. Xia, Y. J. Xiong, B. Lim, S. E. Skrabalak, E. Sara Angew. Chem. Int. Ed., 2009, 48, 60-103.

10 J. Y. Chen, B. Lim, E. P. Lee, Y. N. Xia, Nano Today, 2009, 4, 81-95.

11 S. A. Maier, M. L. Brongersma, P. G. Kik, S. Meltzer, A. A. G. Requicha, H. A. Atwater, Adv. Mater., 2001, 13, 1501-1505.

12 A. N. Shipway, E. Katz, I. Willner, ChemPhysChem, 2000, 1, 18-52.

13 A. W. Sanders, D. A. Routenberg, B. J. Wiley, Y. Xia, E. R. Dufresne, M. A. Reed, Nano Lett., 2006, 6, 1822-1826.

14 T. A. Taton, C. A. Mirkin, R. L. Letsinger, Science, 2000, 289,1757-1760.

15 H. Wang, D. W. Brandl, P. Nordlander, N. J. Halas, Acc. Chem. Res., 2007, 40, 53-62.

16 A. G. Tkachenko, H. Xie, D. Coleman, W. Glomm, J.Ryan, M. F. Anderson, S.

Franzen, D. L. Feldheim, J. Am. Chem. Soc,. 2003, 125, 4700-4701.

17 X. Zhang, M. A. Young, O. Lyandres, R. P. Van Duyne, J. Am. Chem. Soc., 2005, 127, 4484-4489.

18 J. Chen, F. Saeki, B. J. Wiley, H. Cang, M. J. Cobb, Z.-Y. Li, L. Au, H. Zhang, M. B. Kimmey, X. Li, Y. Xia, Nano Lett., 2005, 5, 473-477.

19 X. Yang, S. E. Skrabalak, Z.-Y. Li, Y. Xia, L. V. Wang, Nano Lett., 2007, 7, 37983802 .

20 J. L. West, N. J. Halas, Annu. Rev. Biomed. Eng., 2003, 5, 285-292.

21 S. E. Skrabalak, L. Au, X. Lu, X. Li, Y. Xia, Nanomedicine, 2007, 2, 657-668. 
22 P. Fortina, L. J. Kricka, D. J. Graves, J. Park, T. Hyslop, F. Tam, N. Halas, S. Surrey, S. A. Waldman, Trends Biotechnol., 2007, 25, 145-152.

23 P. K. Jain, X. H. Huang, I. H. El-Sayed, M. A. El-Sayed, Acc. Chem. Res., 2008, 41, 578- 586.

24 M. C. Daniel and D. Astruc, Chem. Rev. 2004, 104, 293-346.

25 K. Saha, S. S. Agasti, C. Kim, X. N. Li, V. M. Rotello, Chem. Rev., 2012, 112, $2739-$ 2779 .

26 W. T. Yu, M. D. Porosoff, J. G. Chen, Chem. Rev., 2012, 112, 5780-5817.

27 D. W. Goodman, Chem. Rev., 1995, 95, 523-536.

28 C. R. Henry, Surf. Sci. Rep., 1998, 31, 235-325.

29 M. Baumer and H. J. Freund, Prog. Surf. Sci., 1998, 61, 127-198.

30 M. Sterrer and H. J. Freund, Catal. Lett., 2013, 143, 375-385.

31 L. N. Lewis, Chem. Rev., 1993, 93, 2693-2730.

32 J. P. Wilcoxon and B. L. Abrams, Chem. Soc. Rev., 2006, 35, 1162-1194.

33 M. A. El-Sayed, Acc. Chem. Res., 2004, 37, 326-333.

34 M. M. Kappes, Chem. Rev., 1988, 88, 369-389.

35 M. T. Reetz, W. Helbig, S. A. Quaiser, U. Stimming, N. Breuer, R. Vogel, Science, 1995, 267, 367-369.

36 H. Lee, S. E. Habas, S. Kweskin, D. Butcher, G. A. Somorjai, P. D. Yang, Angew. Chem., Int. Ed., 2006, 45, 7824-7828.

37 G. A. Somorjai, J. Y. Park, Chem. Soc. Rev., 2008, 37, 2155-2162.

38 Y. Lu, W. Chen, Chem. Soc. Rev., 2012, 41, 3594-3623.

39 M. Turner, V. B. Golovko, O. P. H. Vaughan, P. Abdulkin, A. Berenguer-Murcia, M.

S. Tikhov, B. F. G. Johnson, R. M. Lambert, Nature, 2008, 454, 981-984.

40 F. Baletto, R. Ferrando, Rev. Mod. Phys. 2005, 77, 371-423.

41 R. Ferrando, J. Jellinek, R. L. Johnston, Chem. Rev., 2008, 108, 845-910.

42 I. V. Yudanov, R. Sahnoun, K. M. Neyman, N. Rösch, J. Chem. Phys. 2002, 117, 9887-9896.

43 K. Honkala, A. Hellman, I. N. Remediakis, A. Logadottir, A. Carlsson, S. Dahl, C. H. Christensen, J. K. Norskov, Science, 2005, 307, 555-558. 
44 O. D. Haberlen, S. C. Chung, M. Stener, N. Rosch, J. Chem. Phys. 1997, 106, 51895201.

45 A. Bruix, J. A. Rodriguez, P. J. Ramírez, S. D. Senanayake, J. Evans, J. B. Park, D. Stacchiola, P. Liu, J. Hrbek and F. Illas, J. Am. Chem. Soc., 2012, 134, 8968-8974.

46 S. T. Bromley, I. de P. R. Moreira, K. M. Neyman and F. Illas, Chem. Soc. Rev., 2009, 28, 2657-2670.

47 W. A de Heer, Rev. Mod. Phys. 1993, 65, 611-673.

48 J. Sauer, Chem. Rev. 1989, 89, 1-199.

49 P.S. Bagus and F. Illas, in Encyclopedia of Computational Chemistry, P. v. R. Schleyer, N. L. Allinger, T. Clark, J. Gasteiger, P. A. Kollman, H. F. Schaefer III, P. R. Schreiner, Eds.; John Wiley \& Sons: Chichester, UK, 1998, 4, 2870-2887.

50 G. Wulff, Z. Krist. 1901, 34, 449-530.

51 P. L. Hansen, J. B. Wagner, S. Helveg, J. R. Rostrup-Nielsen, B. S. Clausen, H. Topsoe, Science, 202, 295, 2053-2055.

52 M.C. Desjonqueres, and D. Spanjaard, Concepts in Surface Physics, Springer-Verlag, Belin (1996).

53 A. S. Barnard and P. Zapol, J. Chem. Phys. 2004, 121 4276-4283.

54 C. J. Cramer and D. G. Truhlar, Phys. Chem. Chem. Phys., 2009, 11, 10757-10816.

55 G. Kresse and J. Furthmuller, Phys. Rev. B, 1996, 54, 11169-11186.

56 F. Viñes, F. Illas, K.M. Neyman, J. Phys. Chem. A 2008, 112, 8911-8915.

57 I. V. Yudanov, R. Sahnoun, K. M. Neyman, N. Rösch, J. Hoffmann, S. Schauermann, V. Johánek, H. Unterhalt, G. Rupprechter, J. Libuda, H. -J. Freund, J. Phys. Chem. B 2003, 107, 255-264.

58 F. Viñes, A. Desikusumastuti, Th. Staudt, A. Gorling, J. Libuda, K. M. Neyman, J. Phys. Chem. C 2008, 112, 16539-16549.

59 J. L. C. Fajín, M. N. D. S. Cordeiro, J. R. B. Gomes, F. Illas, J. Chem. Theory Comput. 2012, 8, 1737-1743.

60 I. V. Yudanov, A. Genest, S. Schauermann, H. -J. Freund, N. Rösch, Nano Lett. 2012, 12, 2134-2139. 
61 J. Kleis, J. Greeley, N. A. Romero, V. A. Morozov, H. Falsig, A. H. Larsen, J. Lu, J. J. Mortensen, M. Dułak, K. S. Thygesen, J. K. Nørskov, K. W. Jacobsen, Catal. Lett. 2011, 141, 1067-1071.

62 K. M. Neyman, C. Inntam, A. B. Gordienko, I. V. Yudanov, N. Rösch, J. Chem. Phys., 2005, 122, 174705:1-9.

63 F. Viñes, K. M. Neyman, A. Gorling, J. Phys. Chem. A, 2009, 113, 11963-11973.

64 H. A. Aleksandrov, F. Viñes, W. Ludwig, S. Schauermann, K. M. Neyman, Chem. Eur. J. 2013, 19, 1335-1345.

65 J. L. C. Fajín, A. Bruix, M. N. D. S. Cordeiro, J. R. B. Gomes, F. Illas, J. Chem. Phys. 2012, 137, 034701:1-10.

66 A. Roldán, J. M. Ricart, F. Illas, Theor. Chem. Acc. 2011, 128, 675-681.

67 I. Y. Yudanov, A. V. Matveev, K. M. Neyman, and N. Rösch, J. Am. Chem. Soc. 2008, 130, 9342-9352.

68 F. Viñes, C. Loschen, F. Illas, and K. M. Neyman, J. Catal. 2009, 266, 59-63.

69 A. Roldán, S. González, J. M. Ricart, and F. Illas, ChemPhysChem 2009, 10, 348-351.

70 A. Roldán, J. M. Ricart, and F. Illas, Theor. Chem. Acc. 2011, 128, 675-681.

71 M. Haruta, Nature 2005, 437, 1098.

72 A. Roldán, J. M. Ricart, F. Illas, and G. Pacchioni, Phys. Chem. Chem. Phys. 2010, 12, 10723-10729. 